\title{
The Capacity of Associated Subsequence Retrieval
}

\author{
Behrooz Tahmasebi, Mohammad Ali Maddah-Ali, and Seyed Abolfazl Motahari
}

\begin{abstract}
The objective of a genome-wide association study (GWAS) is to associate subsequences of individuals' genomes to the observable characteristics called phenotypes (e.g., high blood pressure). Motivated by the GWAS problem, in this paper we introduce the information-theoretic problem of associated subsequence retrieval, where a dataset of $N$ (possibly highdimensional) sequences of length $G$, and their corresponding observable (binary) characteristics is given. The sequences are chosen independently and uniformly at random from $\mathcal{X}^{G}$, where $\mathcal{X}$ is a finite alphabet. The observable (binary) characteristic is only related to a specific unknown subsequence of length $L$ of the sequences, called associated subsequence. For each sequence, if the associated subsequence of it belongs to a universal finite set, then it is more likely to display the observable characteristic (i.e., it is more likely that the observable characteristic is one). The goal is to retrieve the associated subsequence using a dataset of $N$ sequences and their observable characteristics. We demonstrate that as the parameters $N, G$, and $L$ grow, a threshold effect appears in the curve of probability of error versus the rate which is defined as $G h(L / G) / N$, where $h(\cdot)$ is the binary entropy function. This effect allows us to define the capacity of associated subsequence retrieval. We develop an achievable scheme and a matching converse for this problem, and thus characterize its capacity in two scenarios: the zero-error-rate and the $\epsilon$-errorrate.
\end{abstract}

Index Terms-Genome-wide association study (GWAS), Shannon theory, threshold effect.

\section{INTRODUCTION}

In a genome-wide association study (GWAS), the ultimate goal is to find common variants within a population which are associated with a complex disease or a given phenotype. This task can be fulfilled by sampling individuals from the population and characterizing their variants and disease status at the same time. Due to the high-dimensionality of genomes and complexity of the association, large number of samples are required to retrieve the associated variants reliably. Fortunately, advances in DNA sequencing and microarray technologies have dramatically decreased the cost of information gathering and made such studies a routine procedure in many centers across the globe.

The first step in a GWAS experiment is to sequence or genotype a set of samples from the genomes population. There exist many tools to reconstruct the genome or identify variants based on raw data, c.f., [2]-[7]. The next step is to infer biological connections between genomic loci and observable characteristics or phenotypes. The objective is to

Behrooz Tahmasebi is with the Department of Electrical Engineering and Computer Science (EECS), Massachusetts Institute of Technology (MIT), Cambridge, MA, USA (e-mail: bzt@mit.edu). Mohammad Ali Maddah-Ali is with the Department of Electrical Engineering, Sharif University of Technology, Tehran 11365, Iran (e-mail: maddah_ali@sharif.edu). Seyed Abolfazl Motahari is with the Department of Computer Engineering, Sharif University of Technology, Tehran 11365, Iran (e-mail: motahari@ sharif.edu).

This paper has been presented at IEEE ISIT 2018 [1]. find a subsequence of length $L$, called associated subsequence, of a genome of length $G$, which correlates with the observed phenotype. In this direction, a fundamental question is how many individuals are required to be sampled to retrieve the associated subsequence reliably.

GWAS has been studied extensively and novel biological results have been discovered (see e.g., [8]-[18]). An important application of GWAS is when the observed phenotype is related to a disease. There are a number of works studying the associated subsequences of diabetes (type I [9] and type II [10]) and various types of cancers [11], e.g., the breast cancer [12] and the prostate cancer [13].

Recently, a number of computational biology problems have been studied from an information-theoretic viewpoint. For example, in the problem of reconstructing a genome from the sequencing reads, information-theoretic limits are characterized in [19], followed by [20]-[26]. Also, a number of authors considered the DNA storage systems, and studied the capacity and coding designs for them in several scenarios [27]-[33]. Haplotype assembly is another example which falls into this category [34], [35].

Motivated by the GWAS problem, in this paper, we introduce an abstract information-theoretic problem of associated subsequence retrieval; see Fig. 1. In this problem, a dataset of $N$ sequences of length $G$ and their corresponding observable (binary) characteristics is given The given sequences are denoted by $\mathbf{x}_{n}, n=1,2, \ldots, N$, and are chosen independently and uniformly at random from $\mathcal{X}^{G}$, for a finite alphabet $\mathcal{X}$. There is a subsequence $\mathbf{s}=\left(s_{1}, s_{2}, \ldots, s_{L}\right)$ of $(1,2, \ldots, G)$, unknown a priori, which is associated with a binary characteristic. In the model, if $\mathbf{x}_{\mathbf{s}}:=\left(x_{s_{1}}, x_{s_{2}}, \ldots, x_{s_{L}}\right)$ equals to one of the $m \in \mathbb{N}$ possible a priori unknown sequences, then the observable characteristic is more likely to be one. The set of $m$ unknown sequences is denoted by $\mathcal{Q}$. Therefore, if $\mathrm{x}_{\mathrm{s}} \notin \mathcal{Q}$ then the corresponding observable characteristic is more likely to be zero. In the considered information-theoretic problem, we would like to study the asymptotic behavior of the minimum sample complexity $N$ such that $\mathbf{s}$ can be reliably identified from the given $N$ sequences and their corresponding observable characteristics.

The main contribution of this paper is to show that there is a threshold effect in the error probability of the associated subsequence retrieval problem. In particular, we define the rate of problem as $\frac{G h(L / G)}{N}$, where $h(\cdot)$ is the binary entropy function. We prove that in the asymptotic regimes, if the rate is less than a threshold, then there exist retrieval schemes with arbitrary low probabilities of error. Conversely, if the rate is above that threshold, then there is no scheme having

${ }^{1}$ In this paper, we assume that the dataset is drawn from one population and it is homogeneous. 


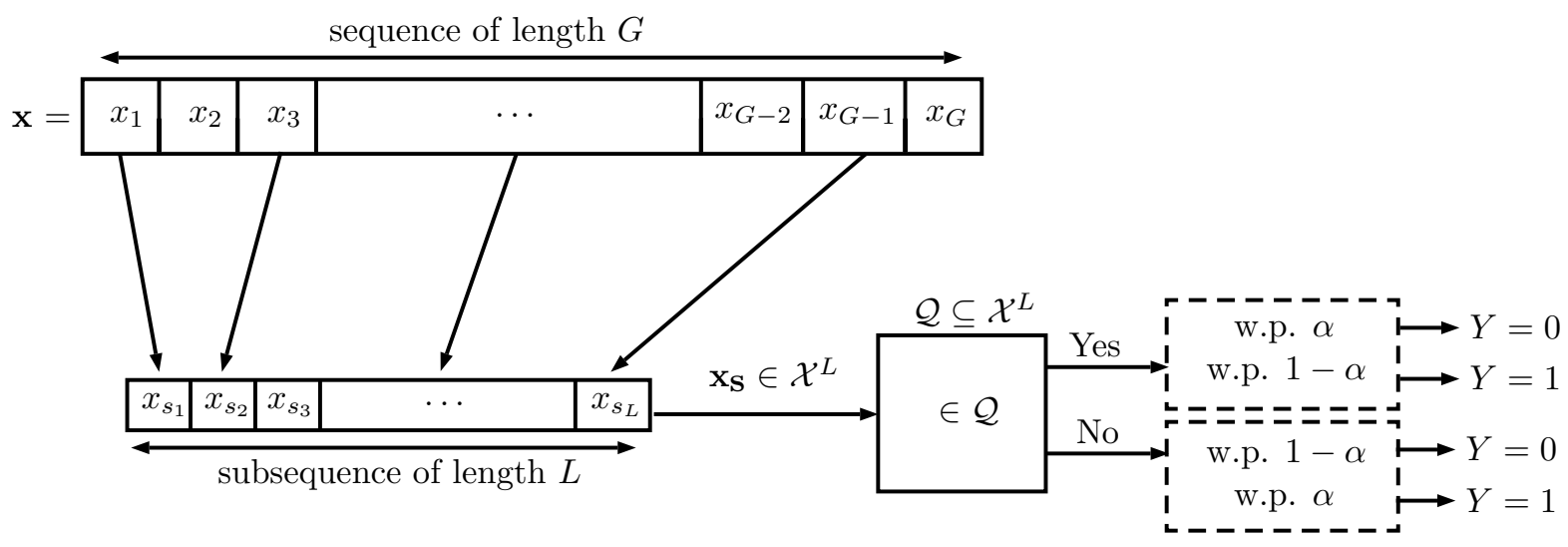

Fig. 1: The problem of associated subsequence retrieval. For each sequence, a specific unknown subsequence of it is chosen, and if the result belongs to a specific universal set $\mathcal{Q}$, then it is more likely to display the characteristic. The main objective is to retrieve the unknown subsequence $\mathbf{s}=\left(s_{1}, s_{2}, \ldots, s_{L}\right)$ from the given $N$ i.i.d. samples, without relying on any prior information about $\mathcal{Q}$.

vanishing probability of error. The threshold is then called the capacity of associated subsequence retrieval, and it is explicitly characterized it in this paper.

In particular, we define two notions of the zero-errorrate and the $\epsilon$-error-rate associated subsequence retrieval. The error-rate is defined as the fraction of incorrectly associated indices in the estimation of the associate subsequence. For the $\epsilon$-error-rate estimation, we are interested in retrieving the associated subsequence with an error-rate of at most $\epsilon$, while for the zero-error-rate estimation, no positive error-rate is acceptable. In the zero-error-rate case, we fully characterize the capacity. The capacity is proven to be a finite positive number, which shows that the scaling of parameters in the definition of rate is reasonable. In the $\epsilon$-error-rate regime, we show that for small enough $\epsilon$, the capacity is the same as the zero-error-rate case. This shows that the two problems of the zero-error-rate and the $\epsilon$-error-rate associated subsequence retrieval are equivalent in the asymptotic regimes.

The rest of the paper is organized as follows. Section II is devoted to the mathematical model and the definition of the capacity. In Section III, the main results of the paper are presented. The proofs are available in Section IV] and Section V] and finally, Section VI concludes the paper.

\section{Problem StATEMENT}

\section{A. Notation}

In this paper, random variables are denoted by capital letters, such as $X$, and their realizations are denoted by lower case letters, such as $x$. But as an exception, we use the capital letters $N, G$ and $L$ to denote the problem's parameters, which are non-random. For a (discrete) random variable $X$, $p_{X}$ denotes its probability mass function. Random probability mass functions are also denoted by capital letters, like $P_{X}$. The sequences are denoted by bold letters, like $\mathbf{x}$, and the random sequences are denoted by capital bold letters, like $\mathbf{X}$.
For any positive integer $G$, let $[G]:=\{1,2, \ldots, G\}$. The $\ell_{p}$ norm of a vector $\mathbf{w} \in \mathbb{R}^{n}$ for $p \geq 1$ is defined as

$$
\|\mathbf{w}\|_{p}:=\left(\sum_{i=1}^{n}\left|w_{i}\right|^{p}\right)^{1 / p}
$$

For a sequence $\mathbf{x}=\left(x_{1}, x_{2}, \ldots, x_{G}\right)$ of length $G$, and a sequence $\mathbf{s}=\left(s_{1}, s_{2}, \ldots, s_{L}\right) \in[G]^{L}$ of length $L$, we define $\mathbf{x}_{\mathbf{s}}:=\left(x_{s_{1}}, x_{s_{2}}, \ldots x_{s_{L}}\right) . \mathbf{x}_{\mathbf{s}}$ is clearly a subsequence of $\mathbf{x}$. Also, we denote the length of a sequence $\mathbf{x}$ by length $(\mathbf{x})$. The base two $\operatorname{logarithm}$ is denoted by $\log ($.$) . For any p \in[0,1]$, the binary entropy function $h:[0,1] \rightarrow[0,1]$ is defined as

$$
h(p):=p \log \left(\frac{1}{p}\right)+(1-p) \log \left(\frac{1}{1-p}\right) .
$$

The mutual information of two discrete random variables $X$ and $Y$ is denoted by $I(X ; Y)$. The distance of two sequences $\mathbf{s}=\left(s_{1}, s_{2}, \ldots, s_{L}\right)$ and $\mathbf{t}=\left(t_{1}, t_{2}, \ldots, t_{L}\right)$ is defined as

$$
\operatorname{dist}(\mathbf{s}, \mathbf{t}):=\left|\left\{s_{1}, s_{2}, \ldots, s_{L}\right\} \triangle\left\{t_{1}, t_{2}, \ldots, t_{L}\right\}\right|,
$$

where $\triangle$ denotes the symmetric difference of sets. The set of all strictly increasing subsequences of length $L$ of $(1,2, \ldots, G)$ is denoted by $\mathcal{S}_{L, G}$. Given a finite set $\mathcal{X}$ and an integer $m$, we define

$$
\mathcal{F}_{L, m}:=\left\{f: \mathcal{X}^{L} \rightarrow\{0,1\}:\left|f^{-1}(1)\right|=m\right\},
$$

where $f^{-1}(1)$ is the set of sequences $\mathbf{x} \in \mathcal{X}^{L}$, such that $f(\mathbf{x})=1$. In other words, $\mathcal{F}_{L, m}$ is the set of functions that exactly map $m$ sequences of $\mathcal{X}^{L}$ to one, and map the others to zero.

\section{B. System Model}

Consider a dataset of $N$ i.i.d. samples $\mathbf{x}_{1}, \mathbf{x}_{2}, \ldots, \mathbf{x}_{N}$, where each sample $\mathbf{x}_{n}$ is chosen uniformly at random from the set of sequences of length $G$ from a finite alphabet $\mathcal{X}$, i.e., $\mathbf{x}_{n} \in \mathcal{X}^{G}$. The observed characteristic of each sample 
$\mathbf{x}_{n}$, denoted by $y_{n}$, can only take two states ${ }^{2}$ denoted by 0 and 1 .

There is a stochastic map $\mathscr{F}: y_{n}=\mathscr{F}\left(\mathbf{x}_{n}\right)$, which associates the $n^{\text {th }}$ sample $\mathbf{x}_{n} \in \mathcal{X}^{G}$ to its observed characteristic $y_{n} \in\{0,1\}$. As depicted in Fig. 1, the map $\mathscr{F}$ is formed as follows: first a specific unknown subsequence of length $L$ of $\mathbf{x}$, denoted by $\mathbf{x}_{\mathbf{s}}=\left(x_{s_{1}}, x_{s_{2}}, \ldots, x_{s_{L}}\right)$, is chosen. Then, $\mathbf{x}_{\mathbf{S}}$ goes through an indication function that outputs 1 if $\mathbf{x}_{\mathbf{s}} \in \mathcal{Q}$, and zero otherwise, for a universal set $\mathcal{Q}$, with size $|\mathcal{Q}|=m, m \in \mathbb{N}$. As shown in Fig. 2, we denote this indication function by $f($.$) . Note that the parameter m$ denotes the number of patterns in the associated subsequence which increase the probability of displaying the characteristic (i.e., the probability of $Y_{n}=1$ ). Then, a Bernoulli random variable is XOR'd with $f\left(\mathbf{x}_{\mathbf{s}}\right)$ and the result is the observed characteristic $y_{n}$. More precisely, $y_{n}=f\left(\mathbf{x}_{n, \mathbf{s}}\right) \oplus Z_{n}$, where $Z_{n}$ is a Bernoulli random variable with parameter $\alpha$. We assume that the additive noises $\left\{Z_{n}\right\}_{n \in \mathbb{N}}$ are independent from the sequences and are independently chosen for different samples. The existence of the additive noise in the problem setup represents the effect of the other factors in the observed characteristic, such as the environmental effects, which are not related to the genome sequences in the GWAS problem. In one extreme, the observed characteristics are highly correlated with the sequences $(\alpha \approx 0)$. In the other extreme, the labels are approximately independent from the sequences $(\alpha \approx 1 / 2)$. We assume in this paper that $\alpha \in[0,1 / 2)$ is given.

In the model, the sequence $\mathbf{s}$ and the deterministic function $f($.$) are unknown but they are the same for all N$ sequences. Throughout this paper, we call $\mathbf{s}$ the associated subsequence, and the main objective is to retrieve s. It is assumed that the parameter $L$ (the length of the associated subsequence) is given. In addition, in this paper, we focus on cases that $L \ll G$. More precisely, we assume that $L / G$ goes to zero, whenever we consider the asymptotic regimes in the paper. This assumption is motivated by what we observed in the GWAS problem. We also assume that the deterministic function $f($.$) is chosen uniformly at random from \mathcal{F}_{L, m}$, defined in (4), for a given positive integer $m$. The associates subsequence $\mathbf{s}$ is selected randomly and uniformly from the set of all strictly increasing sequences of length $L$ with the entries belonging to $[G]$, which is denoted by $\mathcal{S}_{L, G}{ }^{3}$ The entries of the sequence $\mathbf{s}$ represent the sites in each sampled sequence that affect the observed characteristic.

Let us define the parameter $\beta:=\mathbb{P}\left(Y_{n}=1\right)$. Note that $\beta \in(\alpha, 1-\alpha)$ in the proposed data generation model. It is assumed that $\beta$ is known ${ }^{4}$. In this paper, the objective is to estimate $\mathbf{s}$, given $N$ sampled sequences $\left\{\mathbf{x}_{n}\right\}_{n \in[N]}$ and their corresponding observed characteristics $\left\{y_{n}\right\}_{n \in[N]}$. In the

\footnotetext{
${ }^{2}$ For simplicity, in this paper, we only consider binary characteristics. However, the results and proofs of this paper are valid for any characteristic with a finite label set.

${ }^{3}$ We note that because there is no information about $\mathbf{s}$ and $f($.$) in the$ model, we assume that the prior distribution of them is uniform. It is also worth mentioning that the uniform sampling of $\mathbf{s}$ is only needed for the converse proofs. The achievability proofs hold for any prior distribution on the set $\mathcal{S}_{L, G}$. Also, the uniform sampling of $f($.$) is only required for the$ achievability proof and the converse proof holds for any prior on $\mathcal{F}_{L, m}$.

${ }^{4}$ Note that $\beta$ is a constant that only depends on $m, L, \alpha$ and $|\mathcal{X}|$.
}

following, we formally define the algorithms for this purpose.

Definition 1. Algorithm $\mathcal{A}_{(G, L, N, \alpha, \beta, m)}$ is a mapping from the set of all possible input datasets, $\left(\mathcal{X}^{G}\right)^{N} \times\{0,1\}^{N}$, to the set $\mathcal{S}_{L, G}$. When there is no ambiguity, we denote an algorithm by $\mathcal{A}_{G}$ or $\mathcal{A}$. For a dataset $\left(\left\{\mathbf{x}_{n}\right\}_{n \in[N]},\left\{y_{n}\right\}_{n \in[N]}\right)$, $\hat{\mathbf{s}}=\mathcal{A}\left(\left\{\mathbf{x}_{n}\right\}_{n \in[N]},\left\{y_{n}\right\}_{n \in[N]}\right)$ denotes the output of the algorithm.

Next we formally define the error event and also the probability of error for an algorithm.

Definition 2. For a positive $\epsilon$ and an algorithm $\mathcal{A}_{(G, L, N, \alpha, \beta, m)}$, the error event $\mathcal{E}_{\mathcal{A}, \epsilon}$ is defined as $\mathcal{E}_{A, \epsilon}:=\left\{\frac{\operatorname{dist}(\hat{\mathbf{S}}, \mathbf{S})}{L}>\epsilon\right\}$, where $\hat{\mathbf{S}}$ is the output of the algorithm. Also, the worst-case probability of error of an algorithm $\mathcal{A}$ is defined as

$$
P_{\epsilon}^{W C}(\mathcal{A}):=\max _{\mathbf{s} \in \mathcal{S}_{L, G}} \mathbb{P}\left(\mathcal{E}_{\mathcal{A}, \epsilon} \mid \mathbf{S}=\mathbf{s}\right) .
$$

The average probability of error is also defined as $P_{\epsilon}^{A V G}(\mathcal{A}):=$ $\mathbb{P}\left(\mathcal{E}_{\mathcal{A}, \epsilon}\right)$.

Remark 1. Note that the probability measure $\mathbb{P}$ in 5 is defined with respect to the random sequences $\mathbf{X}_{1}, \mathbf{X}_{\mathbf{2}}, \ldots, \mathbf{X}_{N}$, the random noises $Z_{1}, Z_{2}, \ldots, Z_{N}$, the random subsequence $\mathbf{S} \in \mathcal{S}_{L, G}$, and the random function $F(.) \in \mathcal{F}_{L, m}$.

Remark 2. The parameter $\epsilon$ is a threshold for the normalized distance between $\mathbf{s}$ and its estimation $\hat{\mathbf{s}}$. Note that for any algorithm $\mathcal{A}, P_{\epsilon}^{\mathrm{AVG}}(\mathcal{A}) \leq P_{\epsilon}^{\mathrm{WC}}(\mathcal{A})$. Also, by the definition of the error event, if $\epsilon_{1} \geq \epsilon_{2}$ then $\mathcal{E}_{\mathcal{A}, \epsilon_{1}} \subseteq \mathcal{E}_{\mathcal{A}, \epsilon_{2}}$ and thus $P_{\epsilon_{1}}^{\mathrm{WC}}(\mathcal{A}) \leq P_{\epsilon_{2}}^{\mathrm{WC}}(\mathcal{A})$ and $P_{\epsilon_{1}}^{\mathrm{AVG}}(\mathcal{A}) \leq P_{\epsilon_{2}}^{\mathrm{AVG}}(\mathcal{A})$.

In this paper, the goal is to characterize the fundamental limits of the associated subsequence retrieval problem, i.e., the region for the parameters of the problem such that the retrieval of $\mathbf{s}$ is possible. For this purpose, we derive the fundamental limits in two scenarios. First, we study the problem in the zero-error-rate regime, meaning that no positive error-rate is allowed. Second, we study the problem of approximating $\mathbf{s}$ such that a positive error-rate of at most $\epsilon$ is acceptable. In the following definitions, first we define the achievable algorithms and rates and then we define the capacity of problem.

Definition 3. For any positive $\epsilon$, a sequence of algorithms $\left\{\mathcal{A}_{\left(G_{i}, L_{i}, N_{i}, \alpha, \beta, m_{i}\right)}\right\}_{i \in \mathbb{N}^{6}}$, where $\}^{7} m=o(N)$, is said to be $\epsilon$-achievable, if and only if $P_{\epsilon}^{W C}\left(\mathcal{A}_{G_{i}}\right) \rightarrow 0$ as $i \rightarrow \infty$.

Definition 4. A positive real $R$ is said to be an $\epsilon$-achievable rate, if and only if there is an $\epsilon$-achievable sequence of algorithms $\left\{\mathcal{A}_{\left(G_{i}, L_{i}, N_{i}, \alpha, \beta, m_{i}\right)}\right\}_{i \in \mathbb{N}}$, such that $R \leq \frac{G_{i} h\left(L_{i} / G_{i}\right)}{N_{i}}$ for each $i$, where $h($.$) is the binary entropy function.$

${ }^{5}$ In this paper, the required condition to retrieve the associated subsequence is defined based on bounding the probability of having a large errorrate, with respect to a threshold. However, one can see that if the desired condition is defined based on vanishing/bounding the expectation of the error rate, that is $\mathbb{E}\left\{\frac{\operatorname{dist}(\hat{\mathbf{S}}, \mathbf{S})}{L}\right\}$, the same results hold on the capacity of the problem.

${ }^{6}$ Assume that $G_{i}, N_{i}$ and $L_{i}$ are strictly increasing functions of $i$.

${ }^{7}$ By $m=o(N)$, we mean $m_{i} / N_{i} \rightarrow 0$ as $i \rightarrow \infty$. More precisely, this means that the size of the given dataset $N$ is much greater than the number of patterns which increase the probability of displaying the characteristic. 


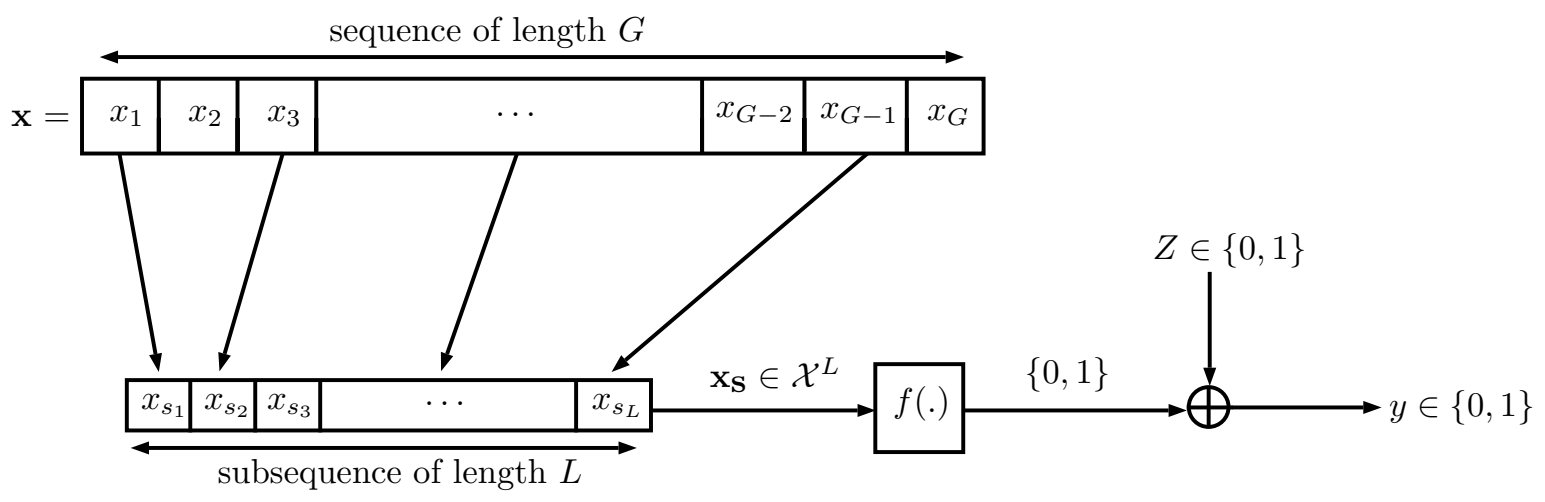

Fig. 2: The system model. For any sequence $\mathbf{x} \in \mathcal{X}^{G}$, a subsequence of it with length $L$ is selected. The function $f($.$) is one$ for $m$ sequences and zero for the other $\left|\mathcal{X}^{L}\right|-m$ sequences. Finally, $y=f\left(\mathbf{x}_{\mathbf{s}}\right) \oplus Z$, where $Z \sim \operatorname{Bern}(\alpha)$, and $\alpha \in[0,0.5)$.

Definition 5. A positive real number $R$ is said to be achievable, if and only if for any positive $\epsilon, R$ is $\epsilon$-achievable.

Now we are ready to define the capacity of the problem.

Definition 6. The zero-error-rate capacity is defined as the supremum of all achievable rates and is denoted by $C(\alpha, \beta)$. Also, for any positive $\epsilon$, the $\epsilon$-capacity is defined as the supremum of all $\epsilon$-achievable rates and is denoted by $C_{\epsilon}(\alpha, \beta)$.

Remark 3. Due to the definitions of the capacities,

$$
C(\alpha, \beta)=\inf _{\epsilon>0} C_{\epsilon}(\alpha, \beta) \leq C_{\epsilon_{1}}(\alpha, \beta) \leq C_{\epsilon_{2}}(\alpha, \beta),
$$

for any positive real numbers $\epsilon_{1}, \epsilon_{2}$, such that $\epsilon_{1} \leq \epsilon_{2}$.

\section{Main Results}

In this section, we state the main results of the paper. In the following theorem, we characterize the capacity of the associated subsequence retrieval $C(\alpha, \beta)$.

Theorem 1. The zero-error-rate capacity of the associated subsequence retrieval is

$$
C(\alpha, \beta)=h(\beta)-h(\alpha),
$$

where $h($.$) is the binary entropy function.$

The achievability proof of the theorem is in Section IV-A. and the proof of the converse can be found in Section IV-B.

Remark 4. Theorem 1 characterizes the capacity of the zeroerror-rate associated subsequence retrieval. This shows that there is a threshold effect in the problem at $G h(L / G) / N$, in the asymptotic regimes. Note that the capacity is strictly positive since $\beta \in(\alpha, 1-\alpha)$.

Remark 5. For the achievability, we examine all the subsequences of length $L$ of the given sequences and choose the one for which the two binary vectors $\left(f\left(\mathbf{x}_{n, \hat{\mathbf{s}}}\right)\right)_{n \in[N]}$ and $\left(y_{n}\right)_{n \in[N]}$ are jointly typical for some $f \in \mathcal{F}_{L, m}$. Note that unlike the channel coding, there is no codebook in this setup and the sequences are produced by nature. This changes the proof techniques. Still, we prove that the probability of error in the proposed scheme goes to zero, using the approximation methods ignoring the dependency among some events and bounding the effect of this assumption.

Remark 6. For the converse, we cannot directly use Fano's inequality due to the definition of the error event. Instead, we develop some inequalities similar to it. The need for this new bound is due to the fact that in this case, if there is an approximation of the associated subsequence with the errorrate of at most $\epsilon$, then we cannot determine it exactly. This fact yields some new terms which appear in the converse proof that are required to be investigated.

We are also interested in characterizing the minimum number of required samples to find an approximation of the associated subsequence with respect to a given positive errorrate $\epsilon$. In the following theorem, we state the result of this paper on the $\epsilon$-error-rate capacity.

Theorem 2. There is a positive $\epsilon_{0} \in(0,1 / 2)$, such that for any $\epsilon \in\left(0, \epsilon_{0}\right)$,

$$
C_{\epsilon}(\alpha, \beta)=h(\beta)-h(\alpha) .
$$

The proof of Theorem 2 can be found in Section $\mathrm{V}$

Remark 7. It may be surprising that the $\epsilon$-capacity is the same as the zero-error-rate capacity. This shows that there is no difference between the approximation of the associated subsequence and the zero-error-rate retrieval in the asymptotic regimes, from the perspective of sample complexity.

Remark 8. To prove Theorem 2, we develop a complementary procedure to convert any algorithm that approximates the associated subsequence, according to an error rate of at most $\epsilon$, to another algorithm that retrieves it with the zero-error-rate condition.

\section{Proof of Theorem 1}

\section{A. Achiveability}

Let $R<h(\beta)-h(\alpha)$ be a positive real number. We aim to prove that $R$ is achievable. In particular, for any positive $\epsilon$, we want to show that $R$ is $\epsilon$-achievable. To this end, let us introduce an algorithm achieving this rate. The algorithm is a jointly typical decoder. First, a few definitions are required. 
Let $F\left(\mathbf{X}_{\mathbf{S}}\right)$ denote the output of the model depicted in Fig. 2, where $\mathbf{X}, \mathbf{S}$, and $F($.$) are uniform random instances from$ $\mathcal{X}^{G}, \mathcal{S}_{L, G}$, and $\mathcal{F}_{L, m}$, respectively. Note that

$$
\begin{aligned}
p_{\left(F\left(\mathbf{X}_{\mathbf{S}}\right), Y\right)}(a, b)= & (\gamma \mathbb{1}\{a=1\}+(1-\gamma) \mathbb{1}\{a=0\}) \times \\
& (\alpha \mathbb{1}\{a \neq b\}+(1-\alpha) \mathbb{1}\{a=b\}),
\end{aligned}
$$

for any $a, b \in\{0,1\}$, where $\gamma:=m /|\mathcal{X}|^{L}$. Also, due to the model,

$$
p_{\left(F\left(\mathbf{X}_{\mathbf{S}}\right), Y\right)}(., .)=p_{\left(F\left(\mathbf{X}_{\mathbf{s}}\right), Y\right)}(., .)=p_{\left(f\left(\mathbf{X}_{\mathbf{s}}\right), Y\right)}(., .),
$$

for any $f(.) \in \mathcal{F}_{L, m}$ and any $\mathbf{s} \in \mathcal{S}_{L, G}$.

Now for any positive $\tau$, let $\mathcal{T}_{\tau}^{N}$ denote the set of all jointly typical binary sequences of length $N$, with respect to $p_{\left(F\left(\mathbf{X}_{\mathbf{S}}\right), Y\right)}$. More precisely,

$$
\begin{aligned}
& \mathcal{T}_{\tau}^{N}:=\left\{(\mathbf{u}, \mathbf{v}) \in\{0,1\}^{N} \times\{0,1\}^{N}:\right. \\
& \left|-\frac{1}{N} \log \left(\prod_{n=1}^{N} p_{F\left(\mathbf{X}_{\mathbf{S}}\right)}\left(u_{n}\right)\right)-H\left(F\left(\mathbf{X}_{\mathbf{S}}\right)\right)\right|<\tau, \\
& \left|-\frac{1}{N} \log \left(\prod_{n=1}^{N} p_{Y}\left(v_{n}\right)\right)-H(Y)\right|<\tau \\
& \left.\left|-\frac{1}{N} \log \left(\prod_{n=1}^{N} p_{\left(F\left(\mathbf{X}_{\mathbf{S}}\right), Y\right)}\left(u_{n}, v_{n}\right)\right)-H\left(F\left(\mathbf{X}_{\mathbf{S}}\right), Y\right)\right|<\tau\right\} .
\end{aligned}
$$

In other words, $\mathcal{T}_{\tau}^{N}$ includes the pairs of binary sequences of length $N$ with empirical entropies $\tau$-close to the true entropies with respect to $p_{\left(F\left(\mathbf{X}_{\mathbf{S}}\right), Y\right)}$ [36]. Note that we only require the parameters $\alpha, \gamma$ to test whether $(\mathbf{u}, \mathbf{v}) \in \mathcal{T}_{\tau}^{N}$ or not 8

The proposed algorithm is as follows.

Algorithm: For the given dataset $\left(\left\{\mathbf{x}_{n}\right\}_{n \in[N]},\left\{y_{n}\right\}_{n \in[N]}\right)$, the algorithm chooses $\hat{\mathbf{s}} \in \mathcal{S}_{L, G}$ with the following property: there is a function $f(.) \in \mathcal{F}_{L, m}$, such that the binary vectors $\left(f\left(\mathbf{x}_{n, \hat{\mathbf{s}}}\right)\right)_{n \in[N]}$ and $\left(y_{n}\right)_{n \in[N]}$ are jointly typical, i.e., $\left(\left(f\left(\mathbf{x}_{n, \hat{\mathbf{s}}}\right)\right)_{n \in[N]},\left(y_{n}\right)_{n \in[N]}\right) \in \mathcal{T}_{\tau}^{N}$. If there are more than one such $\hat{\mathbf{s}}$ with this property, the algorithm chooses one of them at random. If there is no such $\hat{\mathbf{s}}$, the algorithm chooses an element of $\mathcal{S}_{L, G}$ at random. We denote the proposed algorithm by $\mathcal{A}_{G}$.

Remark 9. Note that the typicality decoder also achieves an estimation of the function $f($.$) . But since in this paper we$ only require the asymptotically vanishing error-rate (not the probability $\mathbb{P}(\hat{\mathbf{S}} \neq \mathbf{S}))$, we cannot bound the probability of error for the estimation of $f($.$) in the typicality decoder. We$ also emphasize that the estimation of $f$ can be independently carried out after the estimation of $\mathbf{s}$, and this is known to be a simple learning problem since the VC dimension of the class of functions considered in the paper is bounded $(m \ll N)$.

Analysis of the algorithm: For any fixed positive $\epsilon$, we aim to prove that any $R<h(\beta)-h(\alpha)$ is $\epsilon$-achievable using the proposed algorithm. In particular, we are interested to show

\footnotetext{
${ }^{8}$ We just use the function $F($.$) (or a non-random f($.$) ) and the sequence$ $\mathbf{S}$ (or a non-random $\mathbf{s}$ ) in the indices in notations to remind the definition of them in the proofs.
}

that $P_{\epsilon}^{W C}\left(\mathcal{A}_{G}\right) \rightarrow 0$ as $N, G, L \rightarrow \infty$. In other words, for any $\mathbf{s} \in \mathcal{S}_{L, G}$, we want to prove that the probability $\mathbb{P}\left(\frac{\operatorname{dist}(\hat{\mathbf{S}}, \mathbf{S})}{L}>\right.$ $\epsilon \mid \mathbf{S}=\mathbf{s}$ ) goes to zero in the asymptotic regimes.

Fix an arbitrary $\mathbf{s} \in \mathcal{S}_{L, G}$. Consider the two events $\mathcal{E}_{1}$ and $\mathcal{E}_{2}$ as follows. $\mathcal{E}_{1}$ is the event that the associated subsequence $\mathbf{s}$ does not satisfy the acceptance properties of the algorithm, i.e.,

$$
\mathcal{E}_{1}:=\left\{\left(\left(F\left(\mathbf{X}_{n, \mathbf{S}}\right)\right)_{n \in[N]},\left(Y_{n}\right)_{n \in[N]}\right) \notin \mathcal{T}_{\tau}^{N} \mid \mathbf{S}=\mathbf{s}\right\} .
$$

Also $\mathcal{E}_{2}$ is the event that there exists at least one $\mathbf{t} \in \mathcal{S}_{L, G}$ such that $\mathbf{t}$ satisfies the acceptance properties of the algorithm and $\operatorname{dist}(\mathbf{s}, \mathbf{t})>L \epsilon$. To be more precise, let us define

$$
\mathcal{E}_{\mathbf{t}, g}:=\left\{\left(\left(g\left(\mathbf{X}_{n, \mathbf{t}}\right)\right)_{n \in[N]},\left(Y_{n}\right)_{n \in[N]}\right) \in \mathcal{T}_{\tau}^{N} \mid \mathbf{S}=\mathbf{s}\right\} .
$$

and let

$$
\mathcal{E}_{2}:=\bigcup_{\substack{\mathbf{t} \in \mathcal{S}_{L, G} \\ g(.) \in \mathcal{F}_{L, m} \\ \operatorname{dist}(\mathbf{s}, \mathbf{t})>L \epsilon}} \mathcal{E}_{\mathbf{t}, g} .
$$

Note that to prove the $\epsilon$-achievability of $R$, it just suffices to show that $\mathbb{P}\left(\mathcal{E}_{1} \cup \mathcal{E}_{2}\right) \rightarrow 0$. Using the union of events bound, $\mathbb{P}\left(\mathcal{E}_{1} \cup \mathcal{E}_{2}\right) \leq \mathbb{P}\left(\mathcal{E}_{1}\right)+\mathbb{P}\left(\mathcal{E}_{2}\right)$ and thus it suffices to show that $\mathbb{P}\left(\mathcal{E}_{1}\right)$ and $\mathbb{P}\left(\mathcal{E}_{2}\right)$ vanish in the asymptotic regimes. We note that $\mathbb{P}\left(\mathcal{E}_{1}\right)$ vanishes in the asymptotic regimes, using the law of large numbers. Therefore, to complete the proof, it suffices to show that $\mathbb{P}\left(\mathcal{E}_{2}\right)$ vanishes asymptotically.

Let us state a lemma.

Lemma 1. [37. Theorem 3.5] (Sauer's lemma) For any positive integers $N, m$ and any $\mathbf{t} \in \mathcal{S}_{L, G}$,

$$
\begin{aligned}
\max _{\forall n \in[N]: \mathbf{x}_{n} \in \mathcal{X}^{G}}\left|\left\{\left(g\left(\mathbf{x}_{n, \mathbf{t}}\right)\right)_{n \in[N]} \in\{0,1\}^{N}: g(.) \in \mathcal{F}_{L, m}\right\}\right| \\
\leq \sum_{i=0}^{d}\left(\begin{array}{c}
N \\
i
\end{array}\right),
\end{aligned}
$$

where $d$ is the VC dimension $9^{9}$ of the class of functions $\mathcal{F}_{L, m}$.

Corollary 1. For any positive integers $N \geq m$ and any $\mathbf{t} \in$ $\mathcal{S}_{L, G}$, we have

$$
\begin{aligned}
\max _{\forall n \in[N]: \mathbf{x}_{n} \in \mathcal{X}^{G}} \mid & \left\{\left(g\left(\mathbf{x}_{n, \mathbf{t}}\right)\right)_{n \in[N]} \in\{0,1\}^{N}: g(.) \in \mathcal{F}_{L, m}\right\} \mid \\
\leq & \left(\frac{e N}{m}\right)^{m} .
\end{aligned}
$$

Proof: The VC dimension of the class of functions $\mathcal{F}_{L, m}$ can be upper bounded ${ }^{10}$ by $m$. Therefore, using Lemma 1 and [37. Corollary 3.3], we can establish the desired result.

Corollary 1 relates the parameter $m$ (which is related to the VC dimension of the class of functions considered) to the number of possible observable patterns, given $N$ instances of a function. This bound is used in the asymptotic analysis of the algorithm $\mathcal{A}_{G}$ by the union bound. Note that the condition of Corollary 1 is satisfied, since $m=o(N)$.

\footnotetext{
${ }^{9}$ Vapnik-Chervonenkis dimension.

${ }^{10}$ Indeed, it can be shown that $d=\min \left(m,|\mathcal{X}|^{L}-m\right)$.
} 
Theorem 3. For any positive real numbers $\zeta, \epsilon$, and any $\mathbf{s} \in \mathcal{S}_{L, G}$, with probability $1-o(1)$, the following proposition holds:

For any $\mathbf{t} \in \mathcal{S}_{L, G}$ and any function $g(.) \in \mathcal{F}_{L, m}$, such that $\operatorname{dist}(\mathbf{s}, \mathbf{t})>L \epsilon$, the probability that $\mathbf{t}$ satisfies the acceptance conditions in the proposed algorithm via the function $g($. (i.e., the probability of the event $\mathcal{E}_{\mathbf{t}, g}$ ) is upper bounded by $2^{-N(h(\beta)-h(\alpha)-\zeta)}$.

Proof: See Appendix A

Using Corollary 1, to analyze the algorithm $\mathcal{A}_{G}$, it just suffices to check at most $(e N / m)^{m}$ functions. Let us denote the event in the statement of Theorem 3, which holds with probability $1-o(1)$, by $\mathcal{E}_{3}$. Now we write

$$
\begin{aligned}
\mathbb{P}\left(\mathcal{E}_{2}\right) & =\mathbb{P}\left(\mathcal{E}_{2} \mid \mathcal{E}_{3}^{c}\right) \mathbb{P}\left(\mathcal{E}_{3}^{c}\right)+\mathbb{P}\left(\mathcal{E}_{2} \mid \mathcal{E}_{3}\right) \mathbb{P}\left(\mathcal{E}_{3}\right) \\
& \leq \mathbb{P}\left(\mathcal{E}_{3}^{c}\right)+\mathbb{P}\left(\mathcal{E}_{2} \mid \mathcal{E}_{3}\right) \\
& \leq o(1)+\mathbb{P}\left(\bigcup_{\substack{\mathbf{t} \in \mathcal{S}_{L, G} \\
g(.) \mathcal{F}_{L, m} \\
\operatorname{dist}(\mathbf{s}, \mathbf{t})>L \epsilon}} \mathcal{E}_{\mathbf{t}, g} \mid \mathcal{E}_{3}\right) \\
& \stackrel{(a)}{\leq} o(1)+\sum_{\substack{\mathbf{t} \in \mathcal{S}_{L, G} \\
\operatorname{dist}(\mathbf{s}, \mathbf{t})>L \epsilon}}\left(\frac{e N}{m}\right)^{m} \times 2^{-N(h(\beta)-h(\alpha)-\zeta)} \\
& \leq o(1)+\left(\begin{array}{c}
G \\
L
\end{array}\right) \times 2^{m \log \left(\frac{e N}{m}\right)-N(h(\beta)-h(\alpha)-\zeta)}
\end{aligned}
$$

where (a) follows by Corollary 1 . Theorem 3 , and also the union bound. Using [36. Chapter 11, p. 353], we have $\left(\begin{array}{l}G \\ L\end{array}\right) \leq$ $2^{G h(L / G)}$. Therefore,

$$
\begin{aligned}
\mathbb{P}\left(\mathcal{E}_{2}\right) & \leq o(1)+2^{G h(L / G)+m \log \left(\frac{e N}{m}\right)-N(h(\beta)-h(\alpha)-\zeta)} \\
& =o(1)+2^{N\left(R+\frac{m}{N} \log \left(\frac{e N}{m}\right)-(h(\beta)-h(\alpha)-\zeta)\right)} .
\end{aligned}
$$

We note that $\frac{m}{N} \log \left(\frac{e N}{m}\right) \rightarrow 0$, since $m=o(N)$. Thus, $\mathbb{P}\left(\mathcal{E}_{2}\right)$ vanishes asymptotically if

$$
R-h(\beta)-h(\alpha)-\zeta<0 .
$$

This shows that by choosing small enough $\zeta$, any $R<h(\beta)-$ $h(\alpha)$ is $\epsilon$-achievable. This holds for any positive $\epsilon$ and thus completes the proof.

\section{B. Converse Proof}

In this section, we prove the converse part of Theorem 1 . First we state a lemma.

Lemma 2. For any positive $\epsilon \in(0,1 / 2)$, let $R$ be an $\epsilon$-achievable rate. Then,

$$
R \leq \frac{h(\beta)-h(\alpha)}{1-h(\epsilon)} .
$$

Proof: See appendix B

Now consider an achievable rate $R$. By definition, for any positive $\epsilon, R$ is $\epsilon$-achievable. Using Lemma 2, we conclude that $R \leq \frac{h(\beta)-h(\alpha)}{1-h(\epsilon)}$, for any positive $\epsilon$. Therefore,

$$
R \leq \inf _{\epsilon \in(0,1 / 2)} \frac{h(\beta)-h(\alpha)}{1-h(\epsilon)}=h(\beta)-h(\alpha),
$$

and this completes the proof.

\section{Proof of Theorem 2}

The achievability proof of Theorem 2 directly follows from Theorem 11 Therefore, only the converse proof is required.

Let that $R$ be an $\epsilon$-achievable rate. The goal is to show that $R \leq h(\beta)-h(\alpha)$. First, according to Lemma 2 . $R \leq$ $\frac{h(\beta)-h(\alpha)}{1-h(\epsilon)}$. Consider a sequence of algorithms $\left\{\tilde{\mathcal{A}}_{G_{i}}\right\}_{i \in \mathbb{N}}$, such that $P_{\epsilon}^{W C}\left(\tilde{\mathcal{A}}_{G_{i}}\right) \rightarrow 0$. Define a complementary procedure as follows. Denote the output of the algorithm $\tilde{\mathcal{A}}_{G_{i}}$ by $\tilde{\text { s. Let }}$ $\mathcal{B}_{\tilde{\mathbf{s}}, \epsilon}$ be the ball with radius $L \epsilon$ in $\mathcal{S}_{L, G}$ around $\tilde{\mathbf{s}}$, with respect to the distance dist(.,.). More precisely, we define

$$
\mathcal{B}_{\tilde{\mathbf{s}}, \epsilon}:=\left\{\mathbf{t} \in \mathcal{S}_{L, G}: \operatorname{dist}(\tilde{\mathbf{s}}, \mathbf{t}) \leq L \epsilon\right\} .
$$

Now we apply the proposed algorithm in the achievability proof of Theorem 1 to find $\hat{\mathbf{s}} \in \mathcal{B}_{\tilde{\mathbf{s}}, \epsilon}$ as the estimation of $\mathbf{s}$. More precisely, find the one $\hat{\mathbf{s}} \in \mathcal{B}_{\tilde{\mathbf{s}}, \epsilon}$ such that

$$
\left(\left(f\left(\mathbf{x}_{n, \hat{\mathbf{s}}}\right)\right)_{n \in[N]},\left(y_{n}\right)_{n \in[N]}\right) \in \mathcal{T}_{\tau}^{N},
$$

for some $f(.) \in \mathcal{F}_{L, m}$. If there is not such $\hat{\mathbf{s}}$, choose one of the elements of $\mathcal{B}_{\tilde{\mathbf{s}}, \epsilon}$ randomly. If there is more than one such element, choose one of them randomly.

We aim to prove that the output of this complementary algorithm is a zero-error-rate estimation of $\mathbf{s}$, with high probability. In particular, for any $\epsilon^{\prime} \in(0, \epsilon)$, we show that the output of the complementary procedure has at most $\epsilon^{\prime}$-error-rate, with high probability in the asymptotic regimes. More precisely, we prove that $\mathbb{P}\left(\frac{\text { dist }(\hat{\mathbf{S}}, \mathbf{S})}{L}>\epsilon^{\prime} \mid \mathbf{S}=\mathbf{s}\right) \rightarrow 0$ for any $\epsilon^{\prime} \in(0, \epsilon)$.

Fix a positive $\epsilon^{\prime} \in(0, \epsilon)$. Define two events $\mathcal{E}_{1}$ and $\mathcal{E}_{2}$ similar to the achievability proof of Theorem 1 . More precisely, define

$$
\begin{aligned}
\mathcal{E}_{1}:= & \underbrace{\left\{\left(\left(F\left(\mathbf{X}_{n, \mathbf{S}}\right)\right)_{n \in[N]},\left(Y_{n}\right)_{n \in[N]}\right) \notin \mathcal{T}_{\tau}^{N} \mid \mathbf{S}=\mathbf{s}\right\}}_{\mathcal{E}_{11}} \bigcup \\
& \underbrace{\left\{\mathbf{S} \notin \mathcal{B}_{\tilde{\mathbf{S}}, \epsilon} \mid \mathbf{S}=\mathbf{s}\right\}}_{\mathcal{E}_{12}} .
\end{aligned}
$$

In addition, we define

$$
\begin{aligned}
\mathcal{E}_{\mathbf{t}, g}^{\prime}:= & \underbrace{\left\{\left(\left(g\left(\mathbf{X}_{n, \mathbf{t}}\right)\right)_{n \in[N]},\left(Y_{n}\right)_{n \in[N]}\right) \in \mathcal{T}_{\tau}^{N} \mid \mathbf{S}=\mathbf{s}\right\}}_{\mathcal{E}_{\mathbf{t}, g}} \bigcap \\
& \left\{\mathbf{t} \in \mathcal{B}_{\tilde{\mathbf{S}}, \epsilon} \mid \mathbf{S}=\mathbf{s}\right\},
\end{aligned}
$$

and

$$
\mathcal{E}_{2}^{\prime}:=\bigcup_{\substack{\mathbf{t} \in \mathcal{S}_{L, G} \\ g(.) \in \mathcal{F}_{L, m} \\ \operatorname{dist}(\mathbf{s}, \mathbf{t})>L \epsilon^{\prime}}} \mathcal{E}_{\mathbf{t}, g}^{\prime} .
$$

Note that $\mathcal{E}_{2}^{\prime}$ is defined with respect to the parameter $\epsilon^{\prime}$. To complete the proof, we need to show that $\mathbb{P}\left(\mathcal{E}_{1} \cup \mathcal{E}_{2}^{\prime}\right) \rightarrow 0$. Define

$$
\mathcal{E}_{2}:=\bigcup_{\substack{\mathbf{t} \in \mathcal{B}_{\mathbf{s}, 2 \epsilon} \\ g(.) \in \mathcal{F}_{L, m} \\ \operatorname{dist}(\mathbf{s}, \mathbf{t})>L \epsilon^{\prime}}} \mathcal{E}_{\mathbf{t}, g} .
$$

Claim 1. $\mathcal{E}_{1} \cup \mathcal{E}^{\prime}{ }_{2} \subseteq \mathcal{E}_{1} \cup \mathcal{E}_{2}$. 
Proof: The claim directly follows from the definitions.

As a result, the proof is complete if $\mathbb{P}\left(\mathcal{E}_{1} \cup \mathcal{E}_{2}\right) \rightarrow 0$ or $\mathbb{P}\left(\mathcal{E}_{1}\right), \mathbb{P}\left(\mathcal{E}_{2}\right) \rightarrow 0$. By the assumption, $\operatorname{dist}(\tilde{\mathbf{S}}, \mathbf{s}) \leq L \epsilon$, with high probability. In other words, $\mathbb{P}\left(\mathcal{E}_{12}\right) \rightarrow 0$. By the law of large numbers, $\mathbb{P}\left(\mathcal{E}_{11}\right) \rightarrow 0$. Using the union bound, $\mathbb{P}\left(\mathcal{E}_{1}\right) \rightarrow$ 0 .

To complete the proof, we only need to show that $\mathbb{P}\left(\mathcal{E}_{2}\right)$ goes to zero asymptotically. Similar to the analysis of the proposed algorithm in Theorem 1. using Corollary 1 and Theorem 3, we write ${ }^{11}$

$$
\begin{aligned}
& \mathbb{P}\left(\mathcal{E}_{2}\right) \\
& =\mathbb{P}\left(\mathcal{E}_{2} \mid \mathcal{E}_{3}^{c}\right) \mathbb{P}\left(\mathcal{E}_{3}^{c}\right)+\mathbb{P}\left(\mathcal{E}_{2} \mid \mathcal{E}_{3}\right) \mathbb{P}\left(\mathcal{E}_{3}\right) \\
& \leq \mathbb{P}\left(\mathcal{E}_{3}^{c}\right)+\mathbb{P}\left(\mathcal{E}_{2} \mid \mathcal{E}_{3}\right) \\
& \leq o(1)+\mathbb{P}\left(\bigcup_{\substack{\mathbf{t} \in \mathcal{B}_{\mathbf{s}, 2 \epsilon}(.) \in \mathcal{F}_{L, m} \\
\operatorname{dist}(\mathbf{s}, \mathbf{t})>L \epsilon^{\prime}}} \mathcal{E}_{\mathbf{t}, g} \mid \mathcal{E}_{3}\right) \\
& \leq o(1)+\max _{\mathbf{s} \in \mathcal{S}_{L, G}}\left|\mathcal{B}_{\mathbf{s}, 2 \epsilon}\right| \times\left(\frac{e N}{m}\right)^{m} \times 2^{-N(h(\beta)-h(\alpha)-\zeta)}
\end{aligned}
$$

Now observe that

$$
\begin{aligned}
\log & \left(\left|\mathcal{B}_{\mathbf{s}, 2 \epsilon}\right|\right) \\
& \leq \log \left(\sum_{\ell=0}^{\lfloor 2 L \epsilon\rfloor} \mid\left\{\mathbf{t} \in \mathcal{S}_{L, G}: \operatorname{dist}(\mathbf{s}, \mathbf{t})=\ell \mid\right\}\right) \\
& \leq \log \left(\sum_{\ell=0}^{\lfloor 2 L \epsilon\rfloor}\left(\begin{array}{c}
L \\
\ell
\end{array}\right)\left(\begin{array}{c}
G-L \\
\ell
\end{array}\right)\right) \\
& \leq \log \left((2 L \epsilon+1)\left(\begin{array}{c}
L \\
\lfloor L \epsilon\rfloor
\end{array}\right)\left(\begin{array}{c}
G-L \\
\lfloor 2 L \epsilon\rfloor
\end{array}\right)\right) \\
& (a) \log \left((2 L \epsilon+1) \times 2^{L h(2 \epsilon)} \times 2^{(G-L) h\left(\frac{2 L \epsilon}{G-L}\right)}\right) \\
& \leq \log (2 L \epsilon+1)+L h(2 \epsilon)+(G-L) h\left(\frac{2 L \epsilon}{G-L}\right) \\
& (b) \\
\quad & \log (2 L \epsilon+1)+G h\left(\frac{4 \epsilon L}{G}\right)
\end{aligned}
$$

where (a) follows from [36, Chapter 11, p. 353] and (b) follows from the concavity of the binary entropy function $h($.$) .$ Therefore, using (33) and 40, we write

$$
\begin{aligned}
& \mathbb{P}\left(\mathcal{E}_{2}\right) \\
& \quad \leq o(1)+\max _{\mathbf{s} \in \mathcal{S}_{L, G}}\left|\mathcal{B}_{\mathbf{s}, 2 \epsilon}\right| 2^{m \log \left(\frac{e N}{m}\right)-N(h(\beta)-h(\alpha)-\zeta)} \\
& \quad \leq o(1)+(2 L \epsilon+1) 2^{G h\left(\frac{4 \epsilon L}{G}\right)+m \log \left(\frac{e N}{m}\right)-N(h(\beta)-h(\alpha)-\zeta)} \\
& \quad=o(1)+(2 L \epsilon+1) 2^{N\left(R \frac{h(4 \epsilon L / G)}{h(L / G)}+\frac{m}{N} \log \left(\frac{e N}{m}\right)-(h(\beta)-h(\alpha)-\zeta)\right)} \\
& \quad \stackrel{(a)}{\leq} o(1)+(2 L \epsilon+1) 2^{N\left(R h(2 \epsilon)+\frac{m}{N} \log \left(\frac{e N}{m}\right)-(h(\beta)-h(\alpha)-\zeta)\right)}
\end{aligned}
$$

where (a) follows from Lemma 14

\footnotetext{
${ }^{11}$ Defnine $\mathcal{E}_{3}$ with respect to the parameter $\epsilon^{\prime}$.
}

Now since $\frac{m}{N} \log \left(\frac{e N}{m}\right) \rightarrow 0$, we conclude that $\mathbb{P}\left(\mathcal{E}_{2}\right)$ vanishes asymptotically 12 if

$$
R \leq \frac{h(\beta)-h(\alpha)-\zeta}{h(2 \epsilon)},
$$

for some positive $\zeta$. Let us assume $h(2 \epsilon)+h(\epsilon)<1$. Using Lemma 2, we have

$$
R \leq \frac{h(\beta)-h(\alpha)}{1-h(\epsilon)}<\frac{h(\beta)-h(\alpha)-\zeta}{h(2 \epsilon)},
$$

for small enough $\zeta$. Thus, if $R$ is $\epsilon$-achievable, then it is $\epsilon^{\prime}$-achievable for any $\epsilon^{\prime} \in(0, \epsilon)$. This means that $R$ is achievable. Therefore, using Theorem 1, we conclude that $R \leq h(\beta)-h(\alpha)$ and this completes the proof. Note that there is a positive $\epsilon_{0}$ such that for any $\epsilon<\epsilon_{0}$, we have $h(2 \epsilon)+h(\epsilon)<1$.

Remark 10. Numerical calculation shows that $\epsilon_{0} \approx 0.075$ works for the converse of Theorem 2. However, we do not claim that this is the optimum threshold.

\section{CONCLUSION AND Discussion}

In this paper, the capacity of the associated subsequence retrieval problem, which is inspired by a biological data analysis problem known as genome-wide association study (GWAS), is studied. The fundamental limits of the sample complexity of the problem are derived, for the zero-error-rate and the $\epsilon$-error-rate regimes. In particular, it is shown that the two problems of the $\epsilon$-error-rate and the zero-error-rate associated subsequence retrieval are equivalent.

For the future work, a number of problems can be investigated which are listed below.

- In this paper, it is assumed that the dataset is homogeneous, i.e., the $N$ sampled sequences are associated to only one subsequence. However, it is more realistic to consider the mixed population datasets, where the sampled sequences belong to more than one subpopulation, where each sub-population has a specific associated subsequence and the population origin of the individuals are unknown. This problem has been studied recently in [38].

- Another direction is to consider the non i.i.d. sequences. Although the i.i.d. assumption plays an important role in the proofs of this paper, non i.i.d. sequences, such as stationary Markov models can also be explored for this problem.

- In this paper, it is assumed that the sampling of $N$ sequences is independent from their observed characteristics. However, it is more realistic to consider the nonindependent sampling. For example in the GWAS problem, there exist some cases where a specific phenotype is rare, and hence in the independently sampled datasets, only a a few proportion of the population display the characteristic.

\footnotetext{
${ }^{12}$ The multiplicative factor $2 L \epsilon+1$ does not make any problem, noting that $\frac{\log (L)}{N} \rightarrow 0$ asymptotically. This is due to the fact that by the definition of the problem $N \gg m=\Theta\left(\left|\mathcal{X}^{L}\right|\right)$.
} 
- Another direction for the future work is to consider other notions of the probability of error for the associated subsequence retrieval. For example, it is worth to consider the exact retrieval condition, that is $\mathbb{P}(\hat{\mathbf{S}} \neq \mathbf{S}) \rightarrow 0$. It can be shown that if the function $f($.$) is linear,$ and the sequences are binary, the capacity of associated subsequence retrieval is the same as the one appeared in this paper [39]. However, for the general case, the capacity of the problem is unknown.

- In this paper, it is proved that there is a threshold effect in the cure of the probability of error for associated subsequence retrieval. A remaining problem is to explore how fast this probability of error goes to zero for the rates below the capacity. This problem, which is known as the error exponents problem, is another direction for the further studies.

\section{APPENDIX A \\ PROOF OF THEOREM 3}

To prove Theorem 3 we need a few preliminary definitions and lemmas which are available in the following two subsections.

\section{A. Preliminaries}

In this subsection, we first review some definitions about the divergence measures on probability distributions, as well as their main properties.

Definition 7. Let $f: \mathbb{R}^{\geq 0} \rightarrow \mathbb{R}$ be a convex function, such that $f(1)=0$ and $f(t)$ is strictly convex at $t=1$. Then, the $f$-divergence of any (discrete) probability measures $p_{U}$ and $q_{U}$ on a finite set $\mathcal{U}$ is defined as

$$
D_{f}\left(p_{U} \| q_{U}\right)=\sum_{u \in \mathcal{U}} q_{U}(u) f\left(\frac{p_{U}(u)}{q_{U}(u)}\right) .
$$

We notice that the $f$-divergences satisfy the data processing inequality.

Theorem 4. [40] (Data processing inequality for $f$-divergences). For any (finite) probability measures $p_{U}, q_{U}$ and any channel $p_{V \mid U}$, the following inequality holds.

$$
D_{f}\left(p_{U} \| q_{U}\right) \geq D_{f}\left(p_{U} p_{V \mid U} \| q_{U} p_{V \mid U}\right) .
$$

Note that the function $f(t)=\frac{1}{2}|1-t|$ satisfies the required conditions in Definition 7. It can be shown that in this case, the $f$-divergence reduces to the total variation distance of two probability measures.

In the following definition, we define the $f$-information of two arbitrary (discrete) random variables.

Definition 8. For any (discrete) random variables $U$ and $V$, we define

$$
I_{f}(U ; V):=D_{f}\left(p_{U, V} \| p_{U} p_{V}\right) .
$$

Specifically, for the case of $f(t)=\frac{1}{2}|1-t|$, we can write

$$
I_{f}(U ; V)=\frac{1}{2}\left\|p_{U, V}-p_{U} p_{V}\right\|_{1}
$$

Lemma 3. Consider three random variables $U, V, W$, such that $U-V-W$ is a Markov chain. Then, for any $f$-divergence we have

$$
I_{f}(U ; V) \geq I_{f}(U ; W)
$$

Proof: First we define the following channel

$$
q_{T, W \mid U, V}:=p_{W \mid V} p_{T \mid U}
$$

where $p_{T \mid U}$ is the identity channel, i.e., $T=U$ with probability one. Note that

$$
\begin{aligned}
I_{f}(U ; V) & =D_{f}\left(p_{U, V} \| p_{U} p_{V}\right) \\
& \stackrel{(a)}{\geq} D_{f}\left(p_{U, V} q_{T, W \mid U, V} \| p_{U} p_{V} q_{T, W \mid U, V}\right) \\
& =D_{f}\left(p_{U, V} p_{W \mid V} p_{T \mid U} \| p_{U} p_{V} p_{W \mid V} p_{T \mid U}\right) \\
& \stackrel{(b)}{=} D_{f}\left(p_{U, V} p_{W \mid U, V} p_{T \mid U} \| p_{U} p_{V} p_{W \mid V} p_{T \mid U}\right) \\
& =D_{f}\left(p_{T, W} \| p_{T} p_{W}\right) \\
& =I_{f}(U ; W),
\end{aligned}
$$

which completes the proof. Note that (a) follows from Theorem 4 and (b) follows from the fact that $p_{W \mid U, V}=p_{W \mid V}$.

Corollary 2. Consider random variables $U, V, W, T$, such that $U-V-W-T$ is a Markov chain. Then,

$$
\left\|p_{U, T}-p_{U} p_{T}\right\|_{1} \leq\left\|p_{V, W}-p_{V} p_{W}\right\|_{1} .
$$

Proof: Consider $f(t)=\frac{1}{2}|1-t|$ and use Lemma 3 twice.

Note that $\ell_{1}$ and $\ell_{\infty}$ norms are equivalent.

Lemma 4. For any $\mathrm{w} \in \mathbb{R}^{n}$,

$$
\|\mathbf{w}\|_{\infty} \leq\|\mathbf{w}\|_{1} \leq n\|\mathbf{w}\|_{\infty}
$$

In what follows, we state a few definitions about the dependency of (discrete) random variables.

Definition 9. For any (discrete) random variables $U, V$ and any $\mu \in[1, \infty)$, we write $U \stackrel{\mu}{\perp} V$ if and only if $p_{U V}(u, v) \leq$ $\mu \times p_{U}(u) p_{V}(v)$ for all $u, v$.

Note that for any independent random variables $U, V$, we have $U \stackrel{1}{\perp} V$. Also, if $U \stackrel{\mu}{\perp} V$, then $U \stackrel{\mu^{\prime}}{\perp} V$ for any $\mu^{\prime} \geq \mu$.

The following lemma relates the above definition to the total variation distance.

Lemma 5. Assume that $\left\|p_{U, V}-p_{U} p_{V}\right\|_{1} \leq \epsilon$ for a positive $\epsilon$. Then, for

$$
\mu=1+\frac{\epsilon}{\min _{u} p_{U}(u) \times \min _{v} p_{V}(v)},
$$

we have $U \stackrel{\mu}{\perp} V$.

Proof: First we note that using Lemma 4, we have

$$
\left\|p_{U, V}-p_{U} p_{V}\right\|_{\infty} \leq\left\|p_{U, V}-p_{U} p_{V}\right\|_{1} \leq \epsilon .
$$


Hence, for any $u, v$,

$$
\begin{aligned}
p_{U, V}(u, v) & \leq p_{U}(u) p_{V}(v)+\epsilon \\
& =p_{U}(u) p_{V}(v)\left(1+\frac{\epsilon}{p_{U}(u) p_{V}(v)}\right) \\
& \leq p_{U}(u) p_{V}(v)\left(1+\frac{\epsilon}{\min _{u} p_{U}(u) \times \min _{v} p_{V}(v)}\right) \\
& =\mu \times p_{U}(u) p_{V}(v)
\end{aligned}
$$

which completes the proof.

Lemma 6. For any (discrete) random variables $U, V$ such that $V$ takes values from the set $\{0,1\}$,

$$
\left\|p_{U, V}-p_{U} p_{V}\right\|_{1} \leq 2 \times \max _{u}\left|p_{V \mid U}(1, u)-p_{V}(1)\right| .
$$

Proof: Note that we have

$$
\begin{aligned}
\| p_{U, V}- & p_{U} p_{V} \|_{1}=\sum_{u, v}\left|p_{U, V}(u, v)-p_{U}(u) p_{V}(v)\right| \\
& =\sum_{u, v}\left|p_{U}(u) p_{V \mid U}(v, u)-p_{U}(u) p_{V}(v)\right| \\
& \leq \sum_{u, v} p_{U}(u)\left|p_{V \mid U}(v, u)-p_{V}(v)\right| \\
& =\sum_{u} p_{U}(u)\left|p_{V \mid U}(1, u)-p_{V}(1)\right| \\
& +\sum_{u} p_{U}(u)\left|p_{V \mid U}(0, u)-p_{V}(0)\right| \\
& =\sum_{u} p_{U}(u)\left|p_{V \mid U}(1, u)-p_{V}(1)\right| \\
& +\sum_{u} p_{U}(u)\left|\left(1-p_{V \mid U}(1, u)\right)-\left(1-p_{V}(1)\right)\right| \\
& =2 \times \sum_{u} p_{U}(u)\left|p_{V \mid U}(1, u)-p_{V}(1)\right| \\
& \leq 2 \times \max _{u}\left|p_{V \mid U}(v, u)-p_{V}(v)\right| \\
& \times \sum_{u} p_{U}(u) \\
& 2 \times \max _{u}\left|p_{V \mid U}(1, u)-p_{V}(1)\right| .
\end{aligned}
$$

Concentration inequalities play an important role in the proofs of this paper. Next we state the Hoeffding's inequality.

Lemma 7. [37. Theorem D.1] (Hoeffding's inequality) Let $U_{i}, i \in[n]$, be $n$ i.i.d. random variables taking values in $[a, b]$. Then, for any positive $\epsilon$, we have

$$
\mathbb{P}\left(\left|\frac{U-\mathbb{E}[U]}{n}\right| \geq \epsilon\right) \leq 2 \exp \left(-2 n \epsilon^{2} /(b-a)^{2}\right),
$$

where $U=\sum_{i=1}^{n} U_{i}$.

In what follows, we propose a lemma about the approximation of probabilities.

Lemma 8. Consider $n$ random variables $U_{i}, i \in[n]$, which are distributed according a probability measure $p_{U_{1: n}}$, each over a finite set $\mathcal{U}$. Also consider a discrete random variable $W$ which takes values from a finite set $\mathcal{W}$. Let $w^{*}:=\underset{w \in \mathcal{W}}{\operatorname{argmax}} \mathbb{P}(W=w)$. Consider $n$ random variables $V_{i}$, $i \in[n]$, each takes values from a finite set $\mathcal{V}=\mathcal{U}$, such that $p_{V_{1: n}}=p_{U_{1: n} \mid W=w^{*}}$. All in all, the probability low governing the above random variables factors as

$$
p_{U_{1: n}, V_{1: n}, W}=p_{U_{1: n}} p_{V_{1: n}} p_{W \mid U_{1: n}} .
$$

In addition, consider an arbitrary deterministic function $\psi$ : $\mathcal{U}^{n} \rightarrow\{0,1\}$, and define the events $\mathcal{E}_{1}:=\left\{\psi\left(U_{1: n}\right)=1\right\}$, and $\mathcal{E}_{2}:=\left\{\psi\left(V_{1: n}\right)=1\right\}$. Then,

$$
\mathbb{P}\left(\mathcal{E}_{2}\right) \leq|\mathcal{W}| \times \mathbb{P}\left(\mathcal{E}_{1}\right)
$$

Proof: We write

$$
\begin{aligned}
\mathbb{P}\left(\mathcal{E}_{1}\right) & =\mathbb{E}\left[\psi\left(U_{1: n}\right)\right] \\
& \stackrel{(a)}{=} \mathbb{E}_{W} \mathbb{E}\left[\psi\left(U_{1: n}\right) \mid W\right] \\
& \geq \mathbb{P}\left(W=w^{*}\right) \times \mathbb{E}\left[\psi\left(U_{1: n}\right) \mid W=w^{*}\right] \\
& =\mathbb{P}\left(W=w^{*}\right) \times \mathbb{E}\left[\psi\left(V_{1: n}\right)\right] \\
& \stackrel{(b)}{\geq} \frac{1}{|\mathcal{W}|} \times \mathbb{E}\left[\psi\left(V_{1: n}\right)\right] \\
& =\frac{1}{|\mathcal{W}|} \times \mathbb{P}\left(\mathcal{E}_{2}\right)
\end{aligned}
$$

where (a) follows from the law of iterated expectation and (b) follows from the definition of $w^{*}$.

Lemma 9. For given positive integers $n, m$ such that $n \geq m$, define

$$
\mathcal{Q}_{m}:=\left\{\left(v_{1}, v_{2}, \ldots, v_{n}\right) \in\{0,1\}^{n}: \sum_{i=1}^{n} v_{i}=m\right\} .
$$

Consider $n$ binary random variables $V_{i} \in\{0,1\}, i \in[n]$, which are distributed as

$$
p_{V_{1: n}}\left(v_{1: n}\right)=\frac{1}{\left|\mathcal{Q}_{m}\right|} \times \mathbb{1}\left\{v_{1: n} \in \mathcal{Q}_{m}\right\} .
$$

Then, for any positive $\epsilon$ and any (non-empty) $\mathcal{T} \subseteq[n]$, we have

$$
\mathbb{P}\left(\left|\frac{V-\mathbb{E}[V]}{|\mathcal{T}|}\right| \geq \epsilon\right) \leq 2(n+1) \exp \left(-2|\mathcal{T}| \epsilon^{2}\right),
$$

where $V:=\sum_{i \in \mathcal{T}} V_{i}$.

Proof: The proof is based on Lemma 7 and Lemma 8 Consider $n$ i.i.d. binary random variables $U_{i} \in\{0,1\}, i \in[n]$, such that $p:=\mathbb{P}\left(U_{i}=1\right)=m / n$. Let us define a random variable $W:=\sum_{i \in[n]} U_{i}$. Note that $W$ takes values from the set $\mathcal{W}=\{0,1, \ldots, n\}$ and it is distributed according to a binomial distribution with parameters $n, p$. Note that

$$
\begin{aligned}
w^{*} & =\underset{w \in\{0,1, \ldots, n\}}{\operatorname{argmax}} \mathbb{P}(W=w) \\
& =\underset{w \in\{0,1, \ldots, n\}}{\operatorname{argmax}}\left(\begin{array}{l}
n \\
w
\end{array}\right) \times p^{w} \times(1-p)^{n-w} \\
& \stackrel{(a)}{=} m
\end{aligned}
$$


where (a) follows since $m=n p$ is the mode of $W$.

Now observe that for any $v_{1: n} \in\{0,1\}^{n}$ we have

$$
\begin{aligned}
p_{U_{1: n} \mid W=m} & \left(v_{1: n}\right) \\
& =\frac{\mathbb{P}\left(W=m \mid U_{1: n}=v_{1: n}\right) \times \mathbb{P}\left(U_{1: n}=v_{1: n}\right)}{\mathbb{P}(W=m)} \\
& =\frac{\mathbb{1}\left\{v_{1: n} \in \mathcal{Q}_{m}\right\} \times p^{m} \times(1-p)^{n-m}}{\left(\begin{array}{c}
n \\
m
\end{array}\right) \times p^{m} \times(1-p)^{n-m}} \\
& \stackrel{(a)}{=} \frac{1}{\left(\begin{array}{c}
n \\
m
\end{array}\right)} \times \mathbb{1}\left\{v_{1: n} \in \mathcal{Q}_{m}\right\} \\
& =p_{V_{1: n}}\left(v_{1: n}\right)
\end{aligned}
$$

where (a) follows from the fact that $\left|\mathcal{Q}_{m}\right|=\left(\begin{array}{c}n \\ m\end{array}\right)$. Let us define a function $\psi:\{0,1\}^{n} \rightarrow\{0,1\}$ as follows.

$$
\psi\left(x_{1}, x_{2}, \ldots, x_{n}\right)=\mathbb{1}\left\{\left|\frac{\sum_{i \in \mathcal{T}} x_{i}}{|\mathcal{T}|}-p\right| \geq \epsilon\right\} .
$$

Now observe that the random variables $U_{1: n}, V_{1: n}$ and $W$ and the function $\psi($.$) satisfy the required conditions of Lemma 8$. Hence, if we define $U:=\sum_{i \in \mathcal{T}} U_{i}$, we conclude that

$$
\begin{aligned}
\mathbb{P}\left(\left|\frac{V-\mathbb{E}[V]}{|\mathcal{T}|}\right| \geq \epsilon\right) & \leq|\mathcal{W}| \times \mathbb{P}\left(\left|\frac{U-\mathbb{E}[U]}{|\mathcal{T}|}\right| \geq \epsilon\right) \\
& \stackrel{(a)}{\leq}|\mathcal{W}| \times 2 \exp \left(-2|\mathcal{T}| \epsilon^{2}\right) \\
& \stackrel{(b)}{=} 2(n+1) \exp \left(-2|\mathcal{T}| \epsilon^{2}\right),
\end{aligned}
$$

where (a) follows from Lemma 7 and (b) follows the fact that $\mathcal{W}=\{0,1, \ldots, n\}$. We are done.

The following definitions are about the intersection of the subsequences.

Definition 10. For any $\mathbf{s}, \mathbf{t} \in \mathcal{S}_{L, G}$, define

$$
\operatorname{int}(\mathbf{s}, \mathbf{t}):=\left(w_{1}, w_{2}, \ldots, w_{k}\right) \in[G]^{k}
$$

- such that

- $\left\{w_{1}, w_{2}, \ldots, w_{k}\right\}=\left\{s_{\ell}: \ell \in[L]\right\} \bigcap\left\{t_{\ell}: \ell \in[L]\right\}$,

- $w_{1}<w_{2}<\ldots<w_{k}$.

Definition 11. For any $\mathrm{s} \in \mathcal{S}_{L, G}$, define

$$
\mathcal{I}_{\epsilon}(\mathbf{s}):=\left\{\operatorname{int}(\mathbf{s}, \mathbf{t}) \mid \mathbf{t} \in \mathcal{S}_{L, G}, \operatorname{dist}(\mathbf{s}, \mathbf{t}) \geq L \epsilon\right\} .
$$

Note that we have $\left|\mathcal{I}_{\epsilon}(\mathbf{s})\right| \leq 2^{L}$.

Lemma 10. For any $\mathbf{s}, \mathbf{t} \in \mathcal{S}_{L, G}$, the following statements are equivalent.

- $\operatorname{dist}(\mathbf{s}, \mathbf{t}) \geq L \epsilon$,

- length $(\operatorname{int}(\mathbf{s}, \mathbf{t})) \leq L(1-\epsilon / 2)$.

Proof: Let $\mathcal{S}:=\left\{s_{1}, s_{2}, \ldots, s_{L}\right\}$ and $\mathcal{T}:=$ $\left\{t_{1}, t_{2}, \ldots, t_{L}\right\}$. Note that

$$
\begin{aligned}
\operatorname{dist}(\mathbf{s}, \mathbf{t}) \geq L \epsilon & \Leftrightarrow|\mathcal{S} \cup \mathcal{T}|-|\mathcal{S} \cap \mathcal{T}| \geq L \epsilon \\
& \Leftrightarrow|\mathcal{S}|+|\mathcal{T}|-2|\mathcal{S} \cap \mathcal{T}| \geq L \epsilon \\
& \Leftrightarrow 2 L-2|\mathcal{S} \cap \mathcal{T}| \geq L \epsilon \\
& \Leftrightarrow|\mathcal{S} \cap \mathcal{T}| \leq L(1-\epsilon / 2) \\
& \Leftrightarrow \operatorname{length}(\operatorname{int}(\mathbf{s}, \mathbf{t})) \leq L(1-\epsilon / 2) .
\end{aligned}
$$

\section{B. Preliminaries for Theorem 3}

In this subsection, we present a few lemmas and definitions related to the proof of Theorem 3 .

Definition 12. For any $g(.) \in \mathcal{F}_{L, m}$ and any $\mathbf{t} \in \mathcal{S}_{L, G}$ define a random variable $J_{\mathbf{t}, g}:=g\left(\mathbf{X}_{\mathbf{t}}\right)$. Here $\mathbf{X}$ is a random sequence distributed uniformly over $\mathcal{X}^{G}$.

We note that the probability distribution of $J_{\mathbf{t}, g}$ for any $g(.) \in \mathcal{F}_{L, m}$ and $\mathbf{t}$ is as follows.

$$
p_{J_{\mathbf{t}, g}}(u)= \begin{cases}\gamma & u=1 \\ 1-\gamma & u=0\end{cases}
$$

where $\gamma:=m /|\mathcal{X}|^{L}$ is a parameter. Also, for any function $F($.$) which is chosen randomly and uniformly from the set$ $\mathcal{F}_{L, m}$ and any sequence $\mathbf{S}$ which is chosen randomly and uniformly from the set $\mathcal{S}_{L, G}$, we have the following identity.

$$
p_{J_{\mathbf{t}, g}}=p_{J_{\mathbf{S}, g}}=p_{J_{\mathbf{t}, F}}=p_{J_{\mathbf{S}, F}} .
$$

We note that throughout this section, we have fixed an arbitrary $\mathbf{s} \in \mathcal{S}_{L, G}$ (see Theorem 3 again for more information).

In the following lemma, we aim to show that with probability tending one, $J_{\mathbf{t}, g}$ and $Y$ are (approximately) independent, if $\operatorname{dist}(\mathbf{s}, \mathbf{t}) \geq L \epsilon$. In other words, we want to show that $J_{\mathbf{t}, g} \stackrel{\mu}{\perp} Y$ for some $\mu \rightarrow 1$. Let us clarify this statement in the following lemma.

Lemma 11. For any $g(.) \in \mathcal{F}_{L, m}$, any $\mathbf{t} \in \mathcal{S}_{L, G}$ and also any $\mu \in(1, \infty)$ define the following even ${ }^{13}$

$$
\mathcal{E}_{\mathbf{t}, g}^{\mu}:=\left\{J_{\mathbf{t}, g} \stackrel{\mu}{\perp} Y\right\} .
$$

Note that $Y=J_{\mathbf{s}, F} \oplus Z$, where $Z$ is the additive noise in the model (see Fig. 2). Let us define

$$
\mathcal{E}^{\mu}:=\bigcap_{\substack{\mathbf{t} \in \mathcal{S}_{L, G} \\ \operatorname{dist}(\mathbf{s}, \mathbf{t}) \geq L \epsilon \\ g(.) \in \mathcal{F}_{L, m}}} \mathcal{E}_{\mathbf{t}, g}^{\mu}
$$

Then, $\mathbb{P}\left(\mathcal{E}^{\mu}\right) \rightarrow 1$ for any $\mu \in(1, \infty)$.

Proof: Let us first define

$$
\kappa:=\frac{1}{2}(\mu-1) \times \min (\beta, 1-\beta) \times \min (\gamma, 1-\gamma) .
$$

Assume that $\mathbf{X}$ is chosen randomly and uniformly from the set $\mathcal{X}^{L}$. Let us define the even ${ }^{14}$

$$
\mathcal{E}_{\mathbf{w}, \mathbf{x}^{\prime}}^{\kappa}:=\left\{\left|P_{J_{\mathbf{s}, F}}\right| \mathbf{x}_{\mathbf{w}}=\mathbf{x}^{\prime}(1)-P_{J_{\mathbf{s}, F}}(1) \mid \leq \kappa\right\},
$$

for any $\mathbf{w} \in \mathcal{I}_{\epsilon}(\mathbf{s})$ and any $\mathbf{x}^{\prime} \in \mathcal{X}^{\text {length(w) }}$. Now we need the following two lemmas.

\footnotetext{
${ }^{13}$ Note that the joint distribution $P_{Y, J_{\mathbf{t}, q}}$ depends on the random function $F($.$) . Therefore, it is a random pmf and we denote it by capital letters. As a$ reminder, $F($.$) is uniformly distributed over \mathcal{F}_{L, m}$.

${ }^{14}$ Note that the random choice of the function $F($.$) does not make any$ difference in the distribution of $J_{\mathbf{s}, f}$, i.e., $P_{J_{\mathbf{s}, F}}=p_{J_{\mathbf{s}, f}}$.
} 
Lemma 12.

$$
\begin{aligned}
& \mathcal{E}_{\text {int }}^{\kappa}:=\bigcap_{\substack{\mathbf{t} \in \mathcal{S}_{L, G} \\
\operatorname{dist}(\mathbf{s}, \mathbf{t}) \geq L \epsilon \\
\mathbf{x}^{\prime} \in \mathcal{X}^{\text {length }(\operatorname{int}(\mathbf{s}, \mathbf{t}))}}} \mathcal{E}_{\operatorname{int}(\mathbf{s}, \mathbf{t}), \mathbf{x}^{\prime}}^{\kappa} \\
& \subseteq \mathcal{E}^{\mu}=\bigcap_{\substack{\mathbf{t} \in \mathcal{S}_{L, G} \\
\operatorname{dist}(\mathbf{s}, \mathbf{t}) \geq L \epsilon \\
g(.) \in \mathcal{F}_{L, m}}} \mathcal{E}_{\mathbf{t}, g}^{\mu}
\end{aligned}
$$

Proof: See appendix D

Lemma 13. For any $\mathbf{t} \in \mathcal{S}_{L, G}$, such that $\operatorname{dist}(\mathbf{s}, \mathbf{t}) \geq L \epsilon$, any $g(.) \in \mathcal{F}_{L, m}$, and any $\mathbf{x}^{\prime} \in \mathcal{X}^{\text {length(int }(\mathbf{s}, \mathbf{t}))}$ we have

$$
\mathbb{P}\left(\overline{\mathcal{E}}^{\kappa}{ }_{\text {int }}(\mathbf{s}, \mathbf{t}), \mathbf{x}^{\prime}\right) \leq 2\left(|\mathcal{X}|^{L}+1\right) \exp \left(-2|\mathcal{X}|^{\frac{1}{2} L \epsilon} \kappa^{2}\right) .
$$

Proof: See appendix E

Now using the union bound, we have

$$
\begin{aligned}
& \mathbb{P}\left(\mathcal{E}^{\mu}\right) \stackrel{(a)}{\geq} \mathbb{P}\left(\mathcal{E}_{\text {int }}^{\kappa}\right) \\
& =1-\mathbb{P}\left(\overline{\mathcal{E}_{\text {int }}^{\kappa}}\right) \\
& =1-\mathbb{P}\left(\bigcup_{\substack{\mathbf{t} \in \mathcal{S}_{L, G} \\
\operatorname{dist}(\mathbf{s}, \mathbf{t})>L \epsilon}} \overline{\mathcal{E}^{\kappa}} \operatorname{int}(\mathbf{s}, \mathbf{t}), \mathbf{x}^{\prime}\right) \\
& \mathbf{x}^{\prime} \in \mathcal{X}^{\text {length }(\operatorname{int}(\mathbf{s}, \mathbf{t}))} \\
& =1-\mathbb{P}\left(\bigcup_{\mathbf{w} \in \mathcal{I}_{\epsilon}(\mathbf{s})} \overline{\mathcal{E}^{\kappa}} \mathbf{w}, \mathbf{x}^{\prime}\right) \\
& \mathbf{x}^{\prime} \in \mathcal{X}^{\text {length }(\mathbf{w})} \\
& \geq 1-\sum_{\substack{\mathbf{w} \in \mathcal{I}_{\epsilon}(\mathbf{s}) \\
\mathbf{x}^{\prime} \in \mathcal{X}^{\text {length }(\mathbf{w})}}} \mathbb{P}\left(\overline{\mathcal{E}^{\kappa}} \mathbf{w}, \mathbf{x}^{\prime}\right) \\
& \stackrel{(b)}{\geq} 1-\sum_{\substack{\mathbf{w} \in \mathcal{I}_{\epsilon}(\mathbf{s}) \\
\mathbf{x}^{\prime} \in \mathcal{X}^{\text {length }(\mathbf{w})}}} 2\left(|\mathcal{X}|^{L}+1\right) \exp \left(-2|\mathcal{X}|^{\frac{1}{2} L \epsilon} \kappa^{2}\right) \\
& \stackrel{(c)}{\geq} 1-|\mathcal{X}|^{L} 2^{L+1}\left(|\mathcal{X}|^{L}+1\right) \exp \left(-2|\mathcal{X}|^{\frac{1}{2} L \epsilon} \kappa^{2}\right) \\
& =1-o(1),
\end{aligned}
$$

where (a) follows from Lemma 12, (b) follows from Lemma 13 and (c) follows from the fact that $\left|\mathcal{I}_{\epsilon}(\mathbf{s})\right| \leq 2^{L}$. The proof is thus complete.

\section{Proof of Theorem 3}

Now we are ready to prove Theorem 3 . Fix an arbitrary $\mathbf{s} \in \mathcal{S}_{L, G}$ throughout the proof. Note that

$$
\begin{aligned}
I\left(F\left(\mathbf{X}_{\mathbf{s}}\right) ; Y\right) & =I\left(J_{\mathbf{s}, F} ; Y\right)=H(Y)-H\left(Y \mid F\left(\mathbf{X}_{\mathbf{s}}\right)\right) \\
& =h(\beta)-h(\alpha) .
\end{aligned}
$$

Based on the joint AEP theorem [36, Theorem 7.6.1], if $f\left(\mathbf{X}_{\mathbf{s}}\right)$ is independent of $g\left(\mathbf{X}_{\mathbf{t}}\right)$, for any $f(),. g(.) \in \mathcal{F}_{L, m}$ and any $\mathbf{t} \in \mathcal{S}_{L, G}$ with the normalized distance of at least $\epsilon$ from $\mathbf{s}$, then the desired result is established. However, in the theorem, this condition does not hold. In particular, if two sequences $\mathbf{s}, \mathbf{t}$ intersect, then the independence may not hold. This means that we cannot immediately use the AEP theorem for the proof.
However, we showed that if we choose the function $F(.) \in$ $\mathcal{F}_{L, m}$ uniformly at random, then for sequences like $\mathbf{t}$ that have at least a normalized distance of $\epsilon$ from $\mathbf{s}$, the independence condition holds approximately. We proved this statement in Lemma 11

Now we use similar steps to [36. Theorem 7.6.1] to prove the theorem. Fix an arbitrary $\mu \in(1, \infty)$. Consider the event $\mathcal{E}^{\mu}$ which is defined in Lemma 11. Fix a sequence $\mathbf{t} \in \mathcal{S}_{L, G}$ such that $\operatorname{dist}(\mathbf{s}, \mathbf{t})>L \epsilon$, and a function $g(.) \in \mathcal{F}_{L, m}$. Let $U_{n}:=g\left(\mathbf{X}_{n, \mathbf{t}}\right)$ for any $n \in[N]$.

Note that

$$
\begin{aligned}
\mathbb{P} & \left(\mathcal{E}_{\mathbf{t}, g} \mid \mathcal{E}^{\mu}\right) \\
& =\mathbb{P}\left\{\left(\left(U_{n}\right)_{n \in[N]},\left(Y_{n}\right)_{n \in[N]}\right) \in \mathcal{T}_{\tau}^{N} \mid \mathcal{E}^{\mu}, \mathbf{S}=\mathbf{s}\right\} \\
& =\sum_{\left(u^{N}, y^{N}\right) \in \mathcal{T}_{\tau}^{N}} p_{U^{N}, Y^{N} \mid \mathcal{E}^{\mu}, \mathbf{S}=\mathbf{s}}\left(u^{N}, y^{N}\right) .
\end{aligned}
$$

Using Lemma 11 we conclude that

$$
\begin{aligned}
& \mathbb{P}\left(\mathcal{E}_{\mathbf{t}, g} \mid \mathcal{E}^{\mu}\right)=\sum_{\left(u^{N}, y^{N}\right) \in \mathcal{T}_{\tau}^{N}} p_{U^{N}, Y^{N} \mid \mathcal{E}^{\mu}, \mathbf{S}=\mathbf{s}}\left(u^{N}, y^{N}\right) \\
& \leq \mu^{N} \times \sum_{\left(u^{N}, y^{N}\right) \in \mathcal{T}_{\tau}^{N}} p_{U^{N} \mid \mathcal{E}^{\mu}, \mathbf{S}=\mathbf{s}}\left(u^{N}\right) \times p_{Y^{N} \mid \mathcal{E}^{\mu}, \mathbf{S}=\mathbf{s}}\left(y^{N}\right) \\
& \leq \mu^{N} \times \sum_{\left(u^{N}, y^{N}\right) \in \mathcal{T}_{\tau}^{N}} 2^{-N H\left(g\left(\mathbf{X}_{\mathbf{t}}\right)\right)-N H(Y)+2 N \tau} \\
& \leq \mu^{N} \times 2^{N H\left(g\left(\mathbf{X}_{\mathbf{t}}\right), Y\right)-N H\left(g\left(\mathbf{X}_{\mathbf{t}}\right)\right)-N H(Y)+3 N \tau} \\
& =2^{-N(h(\beta)-h(\alpha)-\log (\mu)-3 \tau)}
\end{aligned}
$$

which completes the proof, if $\mu \in(1, \infty)$ is small enough.

\section{APPENDIX B}

\section{PROOF OF LEMMA 2}

Let us first state some preliminaries. Define $\delta(\epsilon):=$ $\sup _{x \in(0,1 / 2)} \frac{h(2 \epsilon x)}{h(x)}$. It can be shown that $\delta(\epsilon)=h(\epsilon)$ for any $\epsilon \in(0,1 / 2)$. This follows from the following lemma.

Lemma 14. For any $x, y \in[0,1 / 2]$ we have

$$
h(2 x y) \leq h(x) h(y),
$$

where $h($.$) is the binary entropy function.$

Proof: See appendix C

Using Lemma 14, we conclude that

$$
\begin{aligned}
h(\epsilon)=\left.\frac{h(2 \epsilon x)}{h(x)}\right|_{x=1 / 2} & \leq \sup _{x \in(0,1 / 2)} \frac{h(2 \epsilon x)}{h(x)} \\
& \leq \sup _{x \in(0,1 / 2)} h(\epsilon)=h(\epsilon),
\end{aligned}
$$

which shows that $\delta(\epsilon)=h(\epsilon)$. Next we use the function $\delta($. to achieve the desired result.

By the assumption of the lemma, there is a sequence of algorithms $\left\{\mathcal{A}_{\left(G_{i}, L_{i}, N_{i}, \alpha, \beta, m_{i}\right)}\right\}_{i \in \mathbb{N}}$ with rate $R$, such that we have $\lim _{i \rightarrow \infty} P_{\epsilon}^{\mathrm{WC}}\left(\mathcal{A}_{G_{i}}\right)=0$. This implies that $\lim _{i \rightarrow \infty} P_{\epsilon}^{\mathrm{AVG}}\left(\mathcal{A}_{G_{i}}\right)=0$. For a fixed positive integer $i$, let $\mathbf{S}$ be a random sequence that is distributed uniformly over the set $\mathcal{S}_{L_{i}, G_{i}}$. Also let $F($.$) be a random function that is distributed$ 
uniformly over the set $\mathcal{F}_{L_{i}, m_{i}}$. There are $N_{i}$ samples $\mathbf{X}^{N_{i}}$, which are sampled uniformly and independently from the set $|\mathcal{X}|^{G}$, and their corresponding labels $Y^{N_{i}}$, which are generated based on the parameters $\alpha$ and $\beta$. We denote the output of the algorithm $\mathcal{A}_{\left(G_{i}, L_{i}, N_{i}, \alpha, \beta, m_{i}\right)}$ to the dataset $\left(\mathbf{X}^{N_{i}}, \mathbf{Y}^{N_{i}}\right)$ by $\hat{\mathbf{S}}$. Let us define the event $\mathcal{E}_{i}:=\mathcal{E}_{\mathcal{A}_{G_{i}}, \epsilon}=\left\{\frac{\operatorname{dist}(\hat{\mathbf{S}}, \mathbf{S})}{L_{i}}>\epsilon\right\}$ and also let $E_{i}:=\mathbb{1}\left\{\mathcal{E}_{i}\right\}$. Note that $\mathbb{P}\left(\mathcal{E}_{i}\right)=P_{\epsilon}^{\mathrm{AVG}}\left(\mathcal{A}_{G_{i}}\right)$. Hence, we have $\lim _{i \rightarrow \infty} \mathbb{P}\left(\mathcal{E}_{i}\right)=0$.

The proof consists of the following steps.

(i) First we claim that

$$
\begin{aligned}
H(\mathbf{S} \mid \hat{\mathbf{S}}) & \leq 1+\mathbb{P}\left(\mathcal{E}_{i}\right) \log \left(\left(\begin{array}{c}
G_{i} \\
L_{i}
\end{array}\right)\right)+\log \left(L_{i} \epsilon+1\right) \\
& +L_{i} h(\epsilon)+\left(G_{i}-L_{i}\right) h\left(\frac{L_{i} \epsilon}{G_{i}-L_{i}}\right)
\end{aligned}
$$

(ii) Therefore,

$$
\begin{aligned}
& \left.\log \left(\begin{array}{c}
G_{i} \\
L_{i}
\end{array}\right)\right) \\
& \quad=H(\mathbf{S}) \\
& \quad=H(\mathbf{S} \mid \hat{\mathbf{S}})+I(\mathbf{S} ; \hat{\mathbf{S}}) \\
& \quad \leq 1+\mathbb{P}\left(\mathcal{E}_{i}\right) \log \left(\left(\begin{array}{c}
G_{i} \\
L_{i}
\end{array}\right)\right)+\log \left(L_{i} \epsilon+1\right) \\
& \quad+L_{i} h(\epsilon)+\left(G_{i}-L_{i}\right) h\left(\frac{L_{i} \epsilon}{G_{i}-L_{i}}\right)+I(\mathbf{S} ; \hat{\mathbf{S}})
\end{aligned}
$$

(iii) The third step is to show that

$$
I(\mathbf{S} ; \hat{\mathbf{S}}) \leq N_{i} h(\beta)-N_{i} h(\alpha) .
$$

(iv) Combining the above arguments shows that

$$
\begin{aligned}
\log ( & \left.\left(\begin{array}{c}
G_{i} \\
L_{i}
\end{array}\right)\right) \\
& \leq 1+\mathbb{P}\left(\mathcal{E}_{i}\right) \log \left(\left(\begin{array}{c}
G_{i} \\
L_{i}
\end{array}\right)\right)+\log \left(L_{i} \epsilon+1\right) \\
& +L_{i} h(\epsilon)+\left(G_{i}-L_{i}\right) h\left(\frac{\epsilon L_{i}}{G_{i}-L_{i}}\right) \\
& +N_{i} h(\beta)-N_{i} h(\alpha)
\end{aligned}
$$

(v) Then, from [36, Chapter 11, p. 353],

$$
\frac{1}{G_{i}+1} 2^{G_{i} h\left(L_{i} / G_{i}\right)} \leq\left(\begin{array}{c}
G_{i} \\
L_{i}
\end{array}\right) \leq 2^{G_{i} h\left(L_{i} / G_{i}\right)} .
$$

By taking the logarithm from the two sides, we conclude

$$
\begin{aligned}
G_{i} h\left(L_{i} / G_{i}\right)-\log \left(G_{i}+1\right) & \leq \log \left(\left(\begin{array}{c}
G_{i} \\
L_{i}
\end{array}\right)\right) \\
& \leq G_{i} h\left(G_{i} / L_{i}\right) .
\end{aligned}
$$

(vi) Using (150) and (147), we have

$$
\begin{aligned}
& G_{i} h\left(L_{i} / G_{i}\right)-\log \left(G_{i}+1\right) \\
& \leq 1+\mathbb{P}\left(\mathcal{E}_{i}\right) G_{i} h\left(L_{i} / G_{i}\right)+\log \left(L_{i} \epsilon+1\right)+L_{i} h(\epsilon) \\
& +\left(G_{i}-L_{i}\right) h\left(\frac{\epsilon L_{i}}{G_{i}-L_{i}}\right)+N_{i} h(\beta)-N_{i} h(\alpha) .
\end{aligned}
$$

Dividing two sides of 153 by $G_{i} h\left(L_{i} / G_{i}\right)$ results

$$
\begin{aligned}
& 1-\frac{\log \left(G_{i}+1\right)}{G_{i} h\left(L_{i} / G_{i}\right)} \\
& \leq \frac{1}{G_{i} h\left(L_{i} / G_{i}\right)}+\mathbb{P}\left(\mathcal{E}_{i}\right)+\frac{\log \left(L_{i} \epsilon+1\right)}{G_{i} h\left(L_{i} / G_{i}\right)} \\
& +\frac{L_{i}}{G_{i} h\left(L_{i} / G_{i}\right)} h(\epsilon)+\frac{\left(G_{i}-L_{i}\right)}{G_{i} h\left(L_{i} / G_{i}\right)} h\left(\frac{\epsilon L_{i}}{G_{i}-L_{i}}\right) \\
& +\frac{h(\beta)-h(\alpha)}{R} .
\end{aligned}
$$

Using the concavity of the function $h($.$) , we have that$

$$
\frac{L_{i}}{G_{i}} h(\epsilon)+\frac{\left(G_{i}-L_{i}\right)}{G_{i}} h\left(\frac{\epsilon L_{i}}{G_{i}-L_{i}}\right) \leq h\left(2 \epsilon L_{i} / G_{i}\right) .
$$

Hence, we conclude that

$$
\begin{aligned}
1-\frac{\log \left(G_{i}+1\right)}{G_{i} h\left(L_{i} / G_{i}\right)} & \leq \frac{1}{G_{i} h\left(L_{i} / G_{i}\right)}+\mathbb{P}\left(\mathcal{E}_{i}\right) \\
& +\frac{\log \left(L_{i} \epsilon+1\right)}{G_{i} h\left(L_{i} / G_{i}\right)}+\frac{h\left(2 \epsilon L_{i} / G_{i}\right)}{h\left(L_{i} / G_{i}\right)} \\
& +\frac{h(\beta)-h(\alpha)}{R}
\end{aligned}
$$

Applying the inequality $G_{i} h\left(L_{i} / G_{i}\right) \geq L_{i} \log \left(G_{i} / L_{i}\right)$ shows that

$$
\begin{aligned}
1-\frac{\log \left(G_{i}+1\right)}{L_{i} \log \left(G_{i} / L_{i}\right)} & \leq \frac{1}{G_{i} h\left(L_{i} / G_{i}\right)}+\mathbb{P}\left(\mathcal{E}_{i}\right) \\
& +\frac{\log \left(L_{i} \epsilon+1\right)}{L_{i} \log \left(G_{i} / L_{i}\right)}+\frac{h\left(2 \epsilon L_{i} / G_{i}\right)}{h\left(L_{i} / G_{i}\right)} \\
& +\frac{h(\beta)-h(\alpha)}{R} \\
& \stackrel{(a)}{\leq} \frac{1}{G_{i} h\left(L_{i} / G_{i}\right)}+\mathbb{P}\left(\mathcal{E}_{i}\right) \\
& +\frac{\log \left(L_{i} \epsilon+1\right)}{L_{i} \log \left(G_{i} / L_{i}\right)} \\
& +\delta(\epsilon)+\frac{h(\beta)-h(\alpha)}{R}
\end{aligned}
$$

where $(a)$ follows by the definition of the function $\delta($.$) .$

(vii) Finally, we claim that at the limit of $i \rightarrow \infty$, two terms $\frac{\log \left(G_{i}+1\right)}{L_{i} \log \left(G_{i} / L_{i}\right)}$ and $\frac{\log \left(L_{i} \epsilon+1\right)}{L_{i} \log \left(G_{i} / L_{i}\right)}$ go to zero. By letting $i \rightarrow$ $\infty$ in (167), we conclude that

$$
1 \leq \delta(\epsilon)+\frac{h(\beta)-h(\alpha)}{R},
$$

or

$$
R \leq \frac{h(\beta)-h(\alpha)}{1-\delta(\epsilon)}
$$

which completes the proof of the lemma.

In what follows, we prove the above stated claims. In particular, the claims in steps (i),(ii) and (vii) need to be proved. 


\section{A. Proof of (i)}

Note that we have

$$
\begin{aligned}
H\left(E_{i}, \mathbf{S} \mid \hat{\mathbf{S}}\right) & =H(\mathbf{S} \mid \hat{\mathbf{S}})+H\left(E_{i} \mid \mathbf{S}, \hat{\mathbf{S}}\right) \\
& =H\left(E_{i} \mid \hat{\mathbf{S}}\right)+H\left(\mathbf{S} \mid \hat{\mathbf{S}}, E_{i}\right) .
\end{aligned}
$$

Note that $H\left(E_{i} \mid \mathbf{S}, \hat{\mathbf{S}}\right)=0$ and $H\left(E_{i} \mid \hat{\mathbf{S}}\right) \leq 1$. Therefore,

$$
\begin{aligned}
& H(\mathbf{S} \mid \hat{\mathbf{S}}) \\
& \leq 1+H\left(\mathbf{S} \mid \hat{\mathbf{S}}, E_{i}\right) \\
& =1+\mathbb{P}\left(\mathcal{E}_{i}\right) H\left(\mathbf{S} \mid \hat{\mathbf{S}}, E_{i}=1\right)+\left(1-\mathbb{P}\left(\mathcal{E}_{i}\right)\right) H\left(\mathbf{S} \mid \hat{\mathbf{S}}, E_{i}=0\right) \\
& \leq 1+\mathbb{P}\left(\mathcal{E}_{i}\right) H\left(\mathbf{S} \mid \hat{\mathbf{S}}, E_{i}=1\right)+H\left(\mathbf{S} \mid \hat{\mathbf{S}}, E_{i}=0\right) \\
& \stackrel{(a)}{\leq} 1+\mathbb{P}\left(\mathcal{E}_{i}\right) H(\mathbf{S})+H\left(\mathbf{S} \mid \hat{\mathbf{S}}, E_{i}=0\right) \\
& =1+\mathbb{P}\left(\mathcal{E}_{i}\right) \log \left(\left(\begin{array}{c}
G_{i} \\
L_{i}
\end{array}\right)\right)+H\left(\mathbf{S} \mid \hat{\mathbf{S}}, E_{i}=0\right),
\end{aligned}
$$

where (a) follows from the fact that conditioning reduces the entropy. Note that

$$
\begin{aligned}
& H\left(\mathbf{S} \mid \hat{\mathbf{S}}, E_{i}=0\right) \\
& \leq \max _{\hat{\mathbf{s}} \in \mathcal{S}_{L_{i}, G_{i}}} \log \left(\left|\left\{\mathbf{t} \in \mathcal{S}_{L_{i}, G_{i}}: \frac{\operatorname{dist}(\hat{\mathbf{s}}, \mathbf{t})}{L_{i}} \leq \epsilon\right\}\right|\right) \\
& =\max _{\hat{\mathbf{s}} \in \mathcal{S}_{L_{i}, G_{i}}} \log \left(\sum_{\ell=0}^{\left\lfloor L_{i} \epsilon\right\rfloor}\left|\left\{\mathbf{t} \in \mathcal{S}_{L_{i}, G_{i}}: \operatorname{dist}(\hat{\mathbf{s}}, \mathbf{t})=\ell\right\}\right|\right) \\
& =\max _{\hat{\mathbf{s}} \in \mathcal{S}_{L_{i}, G_{i}}} \log \left(\sum_{\ell=0}^{\left\lfloor L_{i} \epsilon\right\rfloor}\left(\begin{array}{c}
L_{i} \\
\ell
\end{array}\right)\left(\begin{array}{c}
G_{i}-L_{i} \\
\ell
\end{array}\right)\right) \\
& \stackrel{(a)}{\leq} \log \left(\left(L_{i} \epsilon+1\right)\left(\begin{array}{c}
L_{i} \\
\left\lfloor L_{i} \epsilon\right\rfloor
\end{array}\right)\left(\begin{array}{c}
G_{i}-L_{i} \\
\left\lfloor L_{i} \epsilon\right\rfloor
\end{array}\right)\right)
\end{aligned}
$$

where (a) follows from the fact that $\epsilon \in(0,1 / 2)$. Using 150$)$ and $(182)$ we conclude

$$
\begin{aligned}
& H\left(\mathbf{S} \mid \hat{\mathbf{S}}, E_{i}=0\right) \\
& \leq \log \left(\left(L_{i} \epsilon+1\right)\left(\begin{array}{c}
L_{i} \\
\left\lfloor L_{i} \epsilon\right\rfloor
\end{array}\right)\left(\begin{array}{c}
G_{i}-L_{i} \\
\left\lfloor L_{i} \epsilon\right\rfloor
\end{array}\right)\right) \\
& \leq \log \left(\left(L_{i} \epsilon+1\right) \times 2^{L_{i} h(\epsilon)} \times 2^{\left(G_{i}-L_{i}\right) h\left(\frac{L_{i} \epsilon}{G_{i}-L_{i}}\right)}\right) \\
& =\log \left(L_{i} \epsilon+1\right)+L_{i} h(\epsilon)+\left(G_{i}-L_{i}\right) h\left(\frac{L_{i} \epsilon}{G_{i}-L_{i}}\right),
\end{aligned}
$$

Combining (177) and (186) results

$$
\begin{aligned}
H(\mathbf{S} \mid \hat{\mathbf{S}}) & \leq 1+\mathbb{P}\left(\mathcal{E}_{i}\right) \log \left(\left(\begin{array}{c}
G_{i} \\
L_{i}
\end{array}\right)\right)+\log \left(L_{i} \epsilon+1\right)+L_{i} h(\epsilon) \\
& +\left(G_{i}-L_{i}\right) h\left(\frac{L_{i} \epsilon}{G_{i}-L_{i}}\right)
\end{aligned}
$$

which completes the proof.

\section{B. Proof of (iii)}

Note that

$$
\begin{aligned}
I(\mathbf{S} ; \hat{\mathbf{S}}) & \stackrel{(a)}{\leq} I\left(\mathbf{S} ; \mathbf{X}^{N_{i}}, Y^{N_{i}}\right) \\
& =I\left(\mathbf{S} ; \mathbf{X}^{N_{i}}\right)+I\left(\mathbf{S} ; Y^{N_{i}} \mid \mathbf{X}^{N_{i}}\right) \\
& \stackrel{(b)}{=} I\left(\mathbf{S} ; Y^{N_{i}} \mid \mathbf{X}^{N_{i}}\right) \\
& =H\left(Y^{N_{i}} \mid \mathbf{X}^{N_{i}}\right)-H\left(Y^{N_{i}} \mid \mathbf{X}^{N_{i}}, \mathbf{S}\right) \\
& \stackrel{(c)}{\leq} N_{i} h(\beta)-H\left(Y^{N_{i}} \mid \mathbf{X}^{N_{i}}, \mathbf{S}\right),
\end{aligned}
$$

where (a) follows from the data processing inequality and the fact that $\mathbf{S} \rightarrow\left(\mathbf{X}^{N_{i}}, Y^{N_{i}}\right) \rightarrow \hat{\mathbf{S}}$ is a Markov Chain, (b) follows from the fact that $\mathbf{S}$ and $\mathbf{X}^{N_{i}}$ are independent random variables and thus $I\left(\mathbf{S} ; \mathbf{X}^{N_{i}}\right)=0$, and (c) follows from the fact that

$$
\begin{aligned}
H\left(Y^{N_{i}} \mid \mathbf{X}^{N_{i}}\right) \leq H\left(Y^{N_{i}}\right)=N_{i} H(Y) & =N_{i} h(\mathbb{P}(Y=1)) \\
& =N_{i} h(\beta)
\end{aligned}
$$

We write

$$
\begin{aligned}
H\left(Y^{N_{i}} \mid \mathbf{X}^{N_{i}}, \mathbf{S}\right) & \stackrel{(a)}{\geq} H\left(Y^{N_{i}} \mid \mathbf{X}^{N_{i}}, \mathbf{S}, F(.)\right) \\
& =H\left(Y^{N_{i}} \mid\left(F\left(\mathbf{X}_{n, \mathbf{S}}\right)\right)_{n \in\left[N_{i}\right]}\right) \\
& \stackrel{(b)}{=} \sum_{n=1}^{N_{i}} H\left(Y_{n} \mid\left(F\left(\mathbf{X}_{n, \mathbf{S}}\right)\right)_{n \in\left[N_{i}\right]}, Y^{n-1}\right) \\
& \stackrel{(c)}{=} N_{i} H\left(Y_{1} \mid F\left(\mathbf{X}_{1, \mathbf{S}}\right)\right) \\
& =N_{i} h(\alpha),
\end{aligned}
$$

where (a) follows by the fact that conditioning reduces the entropy, (b) follows by the telescopic property of the joint entropy and (c) holds because of the memoryless property of the additive noise in the model. Combining (193) and 200) results

$$
I(\mathbf{S} ; \hat{\mathbf{S}}) \leq N_{i} h(\beta)-N_{i} h(\alpha),
$$

which completes the proof.

\section{Proof of (vii)}

To prove the claim, it suffices to only show that $\frac{\log \left(G_{i}+1\right)}{L_{i} \log \left(G_{i} / L_{i}\right)}$ vanishes asymptotically, since $\log \left(G_{i}+1\right) \geq \log \left(L_{i} \epsilon+1\right)$. We write

$$
\begin{aligned}
\frac{\log \left(G_{i}+1\right)}{L_{i} \log \left(G_{i} / L_{i}\right)} & =\frac{\log \left(G_{i}+1\right)}{L_{i} \log \left(G_{i}\right)-L_{i} \log \left(L_{i}\right)} \\
& =\frac{1}{L_{i}\left(\frac{\log \left(G_{i}\right)}{\log \left(G_{i}+1\right)}-\frac{\log \left(L_{i}\right)}{\log \left(G_{i}+1\right)}\right)} .
\end{aligned}
$$


Note that

$$
\begin{aligned}
& L_{i}\left(\frac{\log \left(G_{i}\right)}{\log \left(G_{i}+1\right)}-\frac{\log \left(L_{i}\right)}{\log \left(G_{i}+1\right)}\right) \\
& \geq L_{i}\left(\frac{\log \left(G_{i}\right)}{\log \left(G_{i}+1\right)}-\frac{\log \left(L_{i}\right)}{\log \left(2 L_{i}+1\right)}\right) \\
& =L_{i}\left(\frac{\log \left(G_{i}\right)}{\log \left(G_{i}+1\right)}-\frac{\log \left(2 L_{i}+1\right)+\log \left(\frac{L_{i}}{L_{i}+1 / 2}\right)-1}{\log \left(2 L_{i}+1\right)}\right) \\
& =-L_{i} \frac{\log \left(\left(G_{i}+1\right) / G_{i}\right)}{\log \left(G_{i}+1\right)}+\Theta\left(\frac{L_{i}}{\log \left(L_{i}\right)}\right)
\end{aligned}
$$

Note that $15 L_{i} \frac{\log \left(\left(G_{i}+1\right) / G_{i}\right)}{\log \left(G_{i}+1\right)}=O\left(\frac{L_{i}}{G_{i} \log \left(G_{i}+1\right)}\right)$ and it vanishes asymptotically. Hence, at the limit of $i \rightarrow \infty$, the R.H.S of 207 is $\Theta\left(L_{i} / \log \left(L_{i}\right)\right)$ and so we have $L_{i}\left(\frac{\log \left(G_{i}\right)}{\log \left(G_{i}+1\right)}-\right.$ $\left.\frac{\log \left(L_{i}\right)}{\log \left(G_{i}+1\right)}\right) \rightarrow \infty$ as $i \rightarrow \infty$. Therefore, using 203, we conclude that $\frac{\log \left(G_{i}+1\right)}{L_{i} \log \left(G_{i} / L_{i}\right)} \rightarrow 0$ as $i \rightarrow \infty$ and so the claim is proved.

\section{APPENDIX C}

\section{PROOF OF LEMMA 14}

Let us define $f_{y}(x):=h(x) h(y)-h(2 x y)$ for any $y \in$ $[0,1 / 2]$. Note that $f_{y}(0)=f_{y}(1 / 2)=0$. We aim to prove that $f_{y}($.$) is a non-negative function on the interval [0,1 / 2]$. To complete the proof, it suffices to show that for each $y$, the function $f_{y}($.$) is concave on the interval [0,1 / 2]$.

Note that

$$
\frac{d}{d x} f_{y}(x)=\frac{h(y)}{\ln (2)} \ln \left(\frac{1-x}{x}\right)-\frac{2 y}{\ln (2)} \ln \left(\frac{1-2 x y}{2 x y}\right) .
$$

Now we write

$$
\begin{aligned}
\frac{d^{2}}{d x^{2}} f_{y}(x) & =\frac{h(y)}{\ln (2)} \frac{-1}{x(1-x)}+\frac{4 y^{2}}{\ln (2)} \frac{1}{2 x y(1-2 x y)} \\
& =\frac{1}{x \ln (2)}\left(\frac{-h(y)}{1-x}+\frac{2 y}{1-2 x y}\right) \\
& \leq \frac{1}{x(1-x) \ln (2)}(-h(y)+2 y) \\
& \leq 0
\end{aligned}
$$

which completes the proof, since $h(y) \geq 2 y$ for any $y \in$ $[0,1 / 2]$.

\section{APPENDIX D}

\section{PROOF OF LEMMA 12}

Assume that the event in the L.H.S. of (113) happens. We aim to prove that for any $\mathbf{t} \in \mathcal{S}_{L, G}$, such that $\operatorname{dist}(\mathbf{s}, \mathbf{t}) \geq L \epsilon$,

\footnotetext{
${ }^{15}$ Note that for any constant $c \in(0,1)$, we have $c / G_{i} \leq \ln \left(1+1 / G_{i}\right) \leq$ $1 / G_{i}$ for any large enough $G_{i}$.
}

and any $g(.) \in \mathcal{F}_{L, m}$, the event $\mathcal{E}_{\mathbf{t}, g}^{\mu}$ occurs. We write

$$
\begin{aligned}
& \mathcal{E}_{\mathbf{t}, g}^{\mu}=\left\{J_{\mathbf{t}, g} \perp Y\right\} \\
& \stackrel{(a)}{\supseteq}\left\{\left\|P_{Y, J_{\mathbf{t}, g}}-P_{Y} P_{J_{\mathbf{t}, g}}\right\|_{1}\right. \\
& \left.\quad \leq(\mu-1) \times \min _{y} P_{Y}(y) \times \min _{u} P_{J_{\mathbf{t}, g}}(u)\right\} \\
& =\left\{\left\|P_{Y, J_{\mathbf{t}, g}}-P_{Y} P_{J_{\mathbf{t}, g}}\right\|_{1} \leq 2 \times \kappa\right\} \\
& \stackrel{(b)}{\supseteq}\left\{\left\|P_{\mathbf{X}_{\mathrm{int}(\mathbf{s}, \mathbf{t})}, J_{\mathbf{s}, F}}-P_{\mathbf{X}_{\mathrm{int}(\mathbf{s}, \mathbf{t})}} P_{J_{\mathbf{s}, F}}\right\|_{1} \leq 2 \times \kappa\right\} \\
& \stackrel{(c)}{\supseteq}\left\{\max _{\mathbf{x}^{\prime} \in \mathcal{X}^{\text {length(int (s,t)) }}}\left|P_{J_{\mathbf{s}, F} \mid \mathbf{X}_{\mathrm{int}(\mathbf{s}, \mathbf{t})}=\mathbf{x}^{\prime}}(1)-P_{J_{\mathbf{s}, F}(1)}\right| \leq \kappa\right\} \\
& =\bigcap_{\mathbf{x}^{\prime} \in \mathcal{X}^{\text {length(int }(\mathbf{s}, \mathbf{t}))}}\left\{\left|P_{J_{\mathbf{s}, F} \mid \mathbf{X}_{\mathrm{int}(\mathbf{s}, \mathbf{t})}=\mathbf{x}^{\prime}}(1)-P_{J_{\mathbf{s}, F}(1)}\right| \leq \kappa\right\} \\
& =\bigcap_{\mathbf{x}^{\prime} \in \mathcal{X}^{\text {length(int }(\mathbf{s}, \mathbf{t}))}} \mathcal{E}_{\mathrm{int}(\mathbf{s}, \mathbf{t}), \mathbf{x}^{\prime}}^{\kappa},
\end{aligned}
$$

where (a) follows from Lemma 5, (b) follows from Corollary 2 and the fact that we have the following Markov chain

$$
J_{\mathbf{t}, g}-\mathbf{X}_{\mathbf{t}}-\mathbf{X}_{\mathrm{int}(\mathbf{s}, \mathbf{t})}-\mathbf{X}_{\mathbf{s}}-J_{\mathbf{s}, F}-Y,
$$

and also, (c) follows from Lemma 6. The proof is thus complete.

\section{APPENDIX E \\ PROOF OF LEMMA 13}

Let $\mathbf{X} \in \mathcal{X}^{G}$ be a random sequence which is distributed uniformly over $\mathcal{X}^{G}$. Note that

$$
\begin{aligned}
P_{J_{\mathbf{s}, F} \mid \mathbf{X}_{\mathrm{int}(\mathbf{s}, \mathbf{t})=\mathbf{x}^{\prime}}} & (1) \\
& =\mathbb{E}_{\mathbf{X}}\left[F\left(X_{\mathbf{s}}\right) \mid \mathbf{X}_{\mathrm{int}(\mathbf{s}, \mathbf{t})}=\mathbf{x}^{\prime}, F\right] \\
& \stackrel{(a)}{=} \frac{1}{|\mathcal{X}|^{(L-\operatorname{length}(\operatorname{int}(\mathbf{s}, \mathbf{t})))}} \sum_{\substack{\mathbf{x} \in \mathcal{X}^{G} \\
\mathbf{x}_{\mathrm{int}(\mathbf{s}, \mathbf{t})}=\mathbf{x}^{\prime}}} F\left(\mathbf{x}_{\mathbf{s}}\right),
\end{aligned}
$$

where (a) follows from the fact that

$$
\left|\left\{\mathbf{x} \in \mathcal{X}^{G}: \mathbf{x}_{\operatorname{int}(\mathbf{s}, \mathbf{t})}=\mathbf{x}^{\prime}\right\}\right|=|\mathcal{X}|^{(L-\operatorname{length}(\operatorname{int}(\mathbf{s}, \mathbf{t})))} \text {. }
$$

Now we write

$$
\begin{aligned}
\mathbb{P}\left(\overline{\mathcal{E}}^{\kappa} \operatorname{int}(\mathbf{s}, \mathbf{t}), \mathbf{x}^{\prime}\right) \\
=\mathbb{P}\left(\left|P_{J_{\mathbf{s}, F} \mid \mathbf{X}_{\mathrm{int}(\mathbf{s}, \mathbf{t})}=\mathbf{x}^{\prime}}(1)-P_{J_{\mathbf{s}, F}}(1)\right| \geq \kappa\right) \\
=\mathbb{P}\left(\mid P_{\left.J_{\mathbf{s}, F}\left|\mathbf{X}_{\operatorname{int}(\mathbf{s}, \mathbf{t})=\mathbf{x}^{\prime}}(1)-\gamma\right| \geq \kappa\right)}\right. \\
=\mathbb{P}\left(\left|\frac{1}{|\mathcal{X}|^{(L-\operatorname{length}(\operatorname{int}(\mathbf{s}, \mathbf{t})))}} \sum_{\substack{\mathbf{x} \in \mathcal{X}^{G} \\
\mathbf{x}_{\operatorname{int}(\mathbf{s}, \mathbf{t})=\mathbf{x}^{\prime}}}} F\left(\mathbf{x}_{\mathbf{s}}\right)-\gamma\right| \geq \kappa\right),
\end{aligned}
$$


Now let $\phi: \mathcal{X}^{L} \rightarrow\left[|\mathcal{X}|^{L}\right]$ be a bijection mapping and $n=$ $|\mathcal{X}|^{L}$. Define $n$ random variables $V_{i}=F\left(\phi^{-1}(i)\right), i \in[n]$. Let

$$
\mathcal{T}:=\left\{\phi(\mathbf{x}) \mid \mathbf{x} \in \mathcal{X}^{G}, \mathbf{x}_{\mathrm{int}(\mathbf{s}, \mathbf{t})}=\mathbf{x}^{\prime}\right\} .
$$

Note that $|\mathcal{T}|=|\mathcal{X}|^{(L-\text { length }(\operatorname{int}(\mathbf{s}, \mathbf{t})))}$. Define $V=\sum_{i \in \mathcal{T}} V_{i}$. Note that $\mathbb{E}\left[V_{i}\right]=\gamma$ for any $i$.

Note that the random variables $V_{i}, i \in[n]$, and the set $\mathcal{T} \subseteq$ $[n]$, satisfy the required conditions of Lemma 9 Therefore, using Lemma 9, we conclude that

$$
\begin{aligned}
& \mathbb{P}\left(\overline{\mathcal{E}}^{\kappa} \operatorname{int}(\mathbf{s}, \mathbf{t}), \mathbf{x}^{\prime}\right) \\
& \quad=\mathbb{P}\left(\left|\frac{1}{|\mathcal{X}|^{(L-\text { length(int(s,t)) }}} \sum_{\substack{\mathbf{x} \in \mathcal{X}^{G} \\
\mathbf{x}_{\text {int }(\mathbf{s}, \mathbf{t})=\mathbf{x}^{\prime}}}} F\left(\mathbf{x}_{\mathbf{s}}\right)-\gamma\right| \geq \kappa\right) \\
& \quad=\mathbb{P}\left(\left|\frac{V-\mathbb{E}[V]}{|\mathcal{T}|}\right| \geq \kappa\right) \\
& \quad \leq 2(n+1) \exp \left(-2|\mathcal{T}| \kappa^{2}\right) \\
& \quad=2\left(|\mathcal{X}|^{L}+1\right) \exp \left(-2|\mathcal{X}|^{(L-\operatorname{length}(\operatorname{int}(\mathbf{s}, \mathbf{t})))} \kappa^{2}\right) \\
& \quad \stackrel{(a)}{\leq} 2\left(|\mathcal{X}|^{L}+1\right) \exp \left(-2|\mathcal{X}|^{\frac{1}{2} L \epsilon} \kappa^{2}\right),
\end{aligned}
$$

where (a) follows from Lemma 10 . The proof is thus complete.

\section{REFERENCES}

[1] B. Tahmasebi, M. A. Maddah-Ali, and A. S. Motahari, "Genome-wide association studies: Information theoretic limits of reliable learning," 2018 IEEE International Symposium on Information Theory (ISIT), pp. 2231-22 235, Jun. 2018.

[2] C. Dohm, C. Lottaz, T. Borodina, and H. Himmelbauer, "SHARCGS: A fast and highly accurate short-read assembly algorithm for de novo genomic sequencing," Genome Res., vol. 17, pp. 1697-1706, 2007.

[3] M. S. Waterman and R. M. Idury, "A new algorithm for DNA sequence assembly," J. Comput. Biol., vol. 2, pp. 291-306, 1995.

[4] P. A. Pevzner, H. Tang, and M. S. Waterman, "An eulerian path approach to DNA fragment assembly," Proc. Nat. Acad. Sci. USA, vol. 98, pp. 9748-9753, 2001.

[5] S. Batzoglou, D. B. Jaffe, K. Stanley, J. Butler, S. Gnerre, E. Mauceli, B. Berger, J. P. Mesirov, and E. S. Lander, "Arachne: a whole-genome shotgun assembler," Genome research, vol. 12, no. 1, pp. 177-189, 2002.

[6] G. G. Sutton, O. White, M. D. Adams, , and A. Kerlavage, "TIGR assembler: A new tool for assembling large shotgun sequencing projects," Genome Sci. Technol., vol. 1, pp. 9-19, 1995.

[7] R. L. Warren, G. G. Sutton, S. J. Jones, and R. A. Holt, "Assembling millions of short dna sequences using SSAKE," Bioinformatics, vol. 23, pp. 500-501, 2007.

[8] J. N. Hirschhorn and M. J. Daly, "Genome-wide association studies for common diseases and complex traits," Nature Reviews Genetics, vol. 6, no. 2, pp. 95-108, 2005.

[9] J. C. Barrett, D. G. Clayton, P. Concannon, B. Akolkar, J. D. Cooper, H. A. Erlich, C. Julier, G. Morahan, J. Nerup, C. Nierras et al., "Genome-wide association study and meta-analysis find that over 40 loci affect risk of type 1 diabetes," Nature genetics, vol. 41, no. 6, p. $703,2009$.

[10] K. Hara, H. Fujita, T. A. Johnson, T. Yamauchi, K. Yasuda, M. Horikoshi, C. Peng, C. Hu, R. C. Ma, M. Imamura et al., "Genomewide association study identifies three novel loci for type 2 diabetes," Human molecular genetics, vol. 23, no. 1, pp. 239-246, 2014.

[11] D. F. Easton and R. A. Eeles, "Genome-wide association studies in cancer," Human Molecular Genetics, vol. 17, no. R2, pp. R109-R115, Oct. 2008.

[12] D. F. Easton, K. A. Pooley, A. M. Dunning, P. D. Pharoah, D. Thompson, D. G. Ballinger, J. P. Struewing, J. Morrison, H. Field, R. Luben et al., "Genome-wide association study identifies novel breast cancer susceptibility loci," Nature, vol. 447, no. 7148, pp. 1087-1093, 2007.
[13] G. Thomas, K. B. Jacobs, M. Yeager, P. Kraft, S. Wacholder, N. Orr, K. Yu, N. Chatterjee, R. Welch, A. Hutchinson et al., "Multiple loci identified in a genome-wide association study of prostate cancer," Nature genetics, vol. 40, no. 3, p. 310, 2008.

[14] M. H. Wang, H. J. Cordell, and K. Van Steen, "Statistical methods for genome-wide association studies," in Seminars in cancer biology, vol. 55. Elsevier, 2019, pp. 53-60.

[15] L. T. Elliott, K. Sharp, F. Alfaro-Almagro, S. Shi, K. L. Miller, G. Douaud, J. Marchini, and S. M. Smith, "Genome-wide association studies of brain imaging phenotypes in uk biobank," Nature, vol. 562, no. 7726, pp. 210-216, 2018.

[16] A. I. Young, F. L. Wauthier, and P. Donnelly, "Identifying loci affecting trait variability and detecting interactions in genome-wide association studies," Nature Genetics, vol. 50, pp. 1608-1614, Nov. 2018.

[17] L. Yengo, J. Sidorenko, K. E. Kemper, Z. Zheng, A. R. Wood, M. N. Weedon, T. M. Frayling, J. Hirschhorn, J. Yang, P. M. Visscher et al., "Meta-analysis of genome-wide association studies for height and body mass index in 700000 individuals of european ancestry," Human molecular genetics, vol. 27, no. 20, pp. 3641-3649, 2018.

[18] D. Chang, M. A. Nalls, I. B. Hallgrímsdóttir, J. Hunkapiller, M. Van Der Brug, F. Cai, G. A. Kerchner, G. Ayalon, B. Bingol, M. Sheng et al., "A meta-analysis of genome-wide association studies identifies 17 new parkinson's disease risk loci," Nature genetics, vol. 49, no. 10 , p. 1511, 2017.

[19] A. S. Motahari, G. Bresler, and D. N. C. Tse, "Information theory of DNA shotgun sequencing," IEEE Transactions on Information Theory, vol. 59 , pp. 6273 - 6289, Oct. 2013.

[20] G. Bresler, M. Bresler, and D. Tse, "Optimal assembly for high throughput shotgun sequencing," BMC Bioinformatics, vol. 14, July 2013.

[21] A. Motahari, K. Ramchandran, D. Tse, and N. Ma, "Optimal DNA shotgun sequencing: Noisy reads are as good as noiseless reads," Information Theory Proceedings (ISIT), 2013 IEEE International Symposium on, pp. 1640-1644, 2013

[22] S. Mohajer, A. Motahari, and D. Tse, "Reference-based DNA shotgun sequencing: Information theoretic limits," Information Theory Proceedings (ISIT), 2013 IEEE International Symposium on, pp. 1635-1639, 2013.

[23] I. Shomorony, T. A. Courtade, and D. Tse, "Fundamental limits of genome assembly under an adversarial erasure model," IEEE Transactions on Molecular, Biological and Multi-Scale Communications, vol. 2, pp. 199-208, Dec. 2016.

[24] J. Hui, I. Shomorony, K. Ramchandran, and T. A. Courtade, "Overlapbased genome assembly from variable-length reads," in 2016 IEEE International Symposium on Information Theory (ISIT), July 2016, pp. $1018-1022$

[25] I. Shomorony, G. M. Kamath, F. Xia, T. A. Courtade, and D. N. Tse, "Partial DNA assembly: A rate-distortion perspective," in 2016 IEEE International Symposium on Information Theory (ISIT), July 2016, pp. 1799-1803.

[26] I. Shomorony, T. Courtade, and D. Tse, "Do read errors matter for genome assembly?" in 2015 IEEE International Symposium on Information Theory (ISIT), June 2015, pp. 919-923.

[27] H. M. Kiah, G. J. Puleo, and O. Milenkovic, "Codes for DNA sequence profiles," IEEE Transactions on Information Theory, vol. 62, no. 6, pp. 3125-3146, June 2016.

[28] R. Gabrys, H. M. Kiah, and O. Milenkovic, "Asymmetric lee distance codes for DNA-based storage," IEEE Transactions on Information Theory, vol. 63, no. 8, pp. 4982-4995, Aug 2017.

[29] Z. Chang, J. Chrisnata, M. F. Ezerman, and H. M. Kiah, "Rates of DNA sequence profiles for practical values of read lengths," IEEE Transactions on Information Theory, vol. 63, no. 11, pp. 7166-7177, Nov 2017.

[30] S. M. H. Tabatabaei Yazdi, H. M. Kiah, R. Gabrys, and O. Milenkovic, "Mutually uncorrelated primers for DNA-based data storage," IEEE Transactions on Information Theory, vol. 64, no. 9, pp. 6283-6296, Sep. 2018.

[31] N. Raviv, M. Schwartz, and E. Yaakobi, "Rank-modulation codes for DNA storage with shotgun sequencing," IEEE Transactions on Information Theory, vol. 65, no. 1, pp. 50-64, Jan. 2019

[32] I. Shomorony and R. Heckel, "Capacity results for the noisy shuffling channel," in 2019 IEEE International Symposium on Information Theory (ISIT). IEEE, 2019, pp. 762-766.

[33] R. Heckel, I. Shomorony, K. Ramchandran, and D. N. C. Tse, "Fundamental limits of DNA storage systems," in 2017 IEEE International Symposium on Information Theory (ISIT), June 2017, pp. 3130-3134. 
[34] G. M. Kamath, E. Sasoglu, and D. Tse, "Optimal haplotype assembly from high-throughput mate-pair reads," in 2015 IEEE International Symposium on Information Theory (ISIT), June 2015, pp. 914-918.

[35] H. Si, H. Vikalo, and S. Vishwanath, "Information-theoretic analysis of haplotype assembly," IEEE Transactions on Information Theory, vol. 63 , no. 6, pp. 3468-3479, June 2017.

[36] T. M. Cover and J. A. Thomas, Elements of Information Theory. 2nd ed. New York: John Wiley and Sons, 2006.

[37] M. Mohri, A. Rostamizadeh, and A. Talwalker, Foundations of Machine Learning. The MIT Press, 2012.

[38] B. Tahmasebi, M. A. Maddah-Ali, and S. A. Motahari, "Information theory of mixed population genome-wide association studies," in 2018 IEEE Information Theory Workshop (ITW), Nov. 2018, pp. 1-5.

[39] _ _ "Information theoretic limits of learning of the causal features in a linear model," in 2018 Iran Workshop on Communication and Information Theory (IWCIT), April 2018, pp. 1-6.

[40] F. Liese and I. Vajda, "On divergences and informations in statistics and information theory," IEEE Transactions on Information Theory, vol. 52, no. 10 , pp. 4394-4412, Oct. 2006.

Behrooz Tahmasebi is a Ph.D. student in Electrical Engineering and Computer Science (EECS) at MIT. He received his B.Sc. and M.Sc. degrees from the Department of Electrical Engineering, Sharif University of Technology, Tehran, Iran, in 2016 and 2018, respectively. His research interests include information theory, probability theory, and machine learning.

Mohammad Ali Maddah-Ali (Member, IEEE) received the B.Sc. degree in electrical engineering from the Isfahan University of Technology, the M.A.Sc. degree in electrical engineering from the University of Tehran, and the $\mathrm{PhD}$ degree from the Department of Electrical and Computer Engineering, University of Waterloo, Canada in 2007. From 2007 to 2008, he was with the Wireless Technology Laboratories, Nortel Networks, Ottawa, ON, Canada. From 2008 to 2010, he was a Post-Doctoral Fellow with the Department of Electrical Engineering and Computer Sciences, University of California at Berkeley. Then, he joined Nokia Bell Labs, Holmdel, NJ, USA, as a Communication Research Scientist. Recently, he started working at the Sharif University of Technology as a Faculty Member. He is a recipient of NSERC Postdoctoral Fellowship in 2007, the Best Paper Award from the IEEE International Conference on Communications (ICC) in 2014, the IEEE Communications Society and IEEE Information Theory Society Joint Paper Award in 2015, and the IEEE Information Theory Society Joint Paper Award in 2016.

Seyed Abolfazl Motahari is an assistant professor at Computer Engineering Department of Sharif University of Technology. He received his B.Sc. degree from the Iran University of Science and Technology (IUST), Tehran, in 1999, the M.Sc. degree from Sharif University of Technology, Tehran, in 2001, and the Ph.D. degree from University of Waterloo, Waterloo, Canada, in 2009, all in electrical engineering. From August 2000 to August 2001, he was a Research Scientist with the Advanced Communication Science Research Laboratory, Iran Telecommunication Research Center (ITRC), Tehran. From October 2009 to September 2010, he was a Postdoctoral Fellow with the University of Waterloo, Waterloo. From 2010 to 2013, he was a Postdoctoral Fellow with the Department of Electrical Engineering and Computer Sciences, University of California at Berkeley. His research interests include multiuser information theory and Bioinformatics. He received several awards including Natural Science and Engineering Research Council of Canada (NSERC) PostDoctoral Fellowship. 